\title{
Product Line Design with Quantal Consumer Preferences
}

\author{
Rongyu Wang ${ }^{1, *}$, Xuan Zhao $^{2}$ \\ ${ }^{1}$ Independent Researcher (PhD Economics, University of Edinburgh, 2016), Qingdao, 266072, China \\ ${ }^{2}$ Lazaridis School of Business and Economics, Wilfrid Laurier University, Canada
}

Received May 5, 2020; Revised June 20, 2020; Accepted July 7, 2020

Copyright $\odot 2020$ by authors, all rights reserved. Authors agree that this article remains permanently open access under the terms of the Creative Commons Attribution License 4.0 International License

\begin{abstract}
This paper studies how a firm designs a product line that motivates consumers to deliberate to infer their preferences and buy the product that matches the perceived preferences by deliberation. Some consumers cannot determine their true preferences after deliberation and instead perceive a wrong preference as their own. The statistics describing the distribution of preference perception after deliberation are defined as quantal preferences. By considering the quantal preferences, the firm is only able to design a product line that makes sure the population of consumers with same true preference willingly and truthfully participate the purchasing activity. Our research shows that the equilibrium properties of a screening contract (product line in our context) have been essentially altered by the quantal preferences compared with corresponding properties in a standard screening contract. With quantal preferences, at equilibrium, firm sacrifices part of their profit to reimburse each group of consumers to participate the purchase. Quantal preferences introduce two new incentives into the product line: limiting surplus losses and driving consumer to deliberate the true preference as much as possible. Different incentives can interrelate, e.g. the incentive motivating consumers to truthfully determine their perceived preferences and hence sorting different populations of consumers by the perceived preferences interacting the incentive limiting surplus losses, where deliberation cost adjusts which incentive is stronger. With quantal preferences, high type product can attract low preference consumers. The quality of high type product can be negative. The profit of high type or low type product can be negative, and the surplus of high type or low product can be negative as well. If only one type of product can be produced, it can be the low type product.
\end{abstract}

Keywords Product Line Design, Quantal Preferences, Screening Contract

\section{Introduction}

Consumers usually do not know much about variations of a certain type of product until they are considering making a purchase. Faced with a list of similar products they will carefully compare them and try to determine their preference among them, figuring out which product they like best. This process takes time, and tests the ability of consumers to correctly identify their favorite product. In Guo and Zhang (2012), this thinking process for the purpose of determining one's preference towards a line of products is called deliberation. We have learned from the study of thinking in the field of cognitive psychology that human ability to perform cognitive tasks, for example, paying attention, is usually constrained (Kahneman, 1973).

We can look to the process of selecting consumer finance products for an example. Except for professionals, few people fully understand how financial products (insurance, bonds, securities, etc.) work and how loss and benefit arise from them. Because of this, and because finance products are complex, consumers find it difficult to determine their preferences toward a certain type of products, e.g. investors considering which security to invest in from a list of candidates. As a result, they are still not sure which security is their favorite after a careful thinking, and may choose a wrong security to invest in with the result that it does not produce the expected return. This is a common occurrence in the retail finance industry. Similar examples happen everyday, with consumers regularly making purchasing decisions that are not in their best interests. Due to the human trait of limited thinking, the deliberation process can never be perfect. Mistaken perceptions of one's preferences will happen. In the past decade, many organizations and institutions have been advocating to protect consumers from buying the wrong product through a lack of understanding of the complex nature of the products, especially in consumer finance. Authorities have also proposed much stricter regulations in the industry to protect consumers' interests when consuming sophisticated 
products (World Bank Group, 2017; OECD, 2010). Regulation can protect consumers' interests in the short term, but it can reduce operations and over the long term weaken services and competition. Therefore, it is desirable for companies to be productive in protecting consumer interests rather than passively protecting consumers due to the requirements from regulation authorities.

One way for the firm to protect consumer interests, while securing its own benefits, is to take into account during product design that some consumers may choose wrongly due to a mistakenly perceived preference. In this paper, we propose such a mechanism in the context of designing a product line. Consumers have either high valuation or low valuation towards a type of product, e.g. the mutual fund. High valuation consumers prefer a fund that provides high yield with reasonable risk, while the low valuation consumers prefer a fund that provides high security with a reasonable yield. However, consumers' limited cognitive ability constraints their understanding of the products, and they may be unable to determine their preference from list of funds. They may not understand the fund policy, no matter how many times they read the documentation. However, their understanding of the products after careful readings can be improved and their choices become closer to their true preferences, which is the best status they can achieve. Due to the imperfect understanding of their own preferences, however, some consumers will choose a product consistent with a mistaken preference. A firm should consider this fact when it designs the two funds. According to the results from our product line design model, designing funds with this in mind can benefit the firm. Although its aggregate profit is smaller than the counterpart when it ignores consumers who perceive a wrong preference after deliberation, designing products in this way can limit the surplus losses for consumers who perceive a wrong preference and hence buy the fund inconsistent with their true preferences.

The status that after deliberation humans may not correctly perceive their preferences can be summarized by a set of statistics. As the result of humans' limited cognitive ability in thinking, the statistics describe an individual's belief on his preference after deliberation. We define such statistics by quantal preferences. According to the law of large numbers, the quantal preferences also describe the proportion of people who have correctly or incorrectly perceived their preferences after deliberation. The statistics for representing quantal preferences can be usually found from experimental data or market survey on consumer preferences. In the next section, we review the literature that discovered the phenomenon that humans' stated preferences may not be their true preferences. This phenomenon was discovered and formally reported from experimental data, especially from the experiments by Harless and Camerer (1994) and Hey and Orme (1994).

In this paper, we consider how a firm can design a product line taking into consideration the quantal consumer preferences, i.e. considering the changes that quantal consumer preferences bring to a product line relative to its counterpart designed without considering consumers' wrong perceptions. The product line designed without considering consumer perceiving a wrong preference is studied by Guo and Zhang (2012). In Guo and Zhang (2012), consumers have to deliberate to find out their preferences, and then choose the product matching their preferences from the product line. After deliberation, consumers can always accurately find out their true preferences. Therefore, consumers are fully informed about their own preferences, and the product line design problem in Guo and Zhang (2012) can be dealt with as designing a screening contract. There are some regular properties for screening contracts, and the equilibrium product line in Guo and Zhang (2012) also follows these properties in a product line context. Since most screening contracts are analyzed in a binary-type framework, our model, although it is studied in a product line design context, has general implications for the theory of screening contract.

The quantal consumer preferences introduce state nonverifiability in the product line design problem. Before consumers finish using the product, they do not know what their underlying preferences are and whether their perceived preferences are the same as their true preferences. When they decide to participate the purchasing activity, their decisions are based on their perceived preferences, a phenomenon that is usual and echoes our daily behavior. If consumers always hold an uncertain belief about their true preferences and swing between different preferences, it is hard to expect that they will make a decision. Besides, experiment findings have shown that when consumers are uncertain about their preferences, expected utility theory can be violated (Harless and Camerer, 1994; Hey and Orme, 1994). Therefore, in the interpretation of our product line design, we regard it as a population-oriented contract design, not a contract designed at individual level. It features maximizing the aggregate surplus for a group of consumers, not maximizing a single consumer's expected surplus.

Analyzing the product line at equilibrium, we note that in the product line design with quantal preferences, consumers who correctly perceive their preferences after deliberation can get more surplus than in the fully informative setting studied by Guo and Zhang (2012). The additional surplus ensures the population of consumers of the same preference type possess a non-negative aggregate surplus so that the entire population of the same true preference is willing to participate the purchasing activity. The firm, as the product line designer, will sacrifice part of its profit relative to what it could have earned in the fully informative case to ensure each population of consumers is willing to participate the purchasing activity.

After incorporating the quantal preferences into the product line design, the approaches to solving the product line design problem, which in principle still follows the manner for a binary type screening contract design, become flexible. The approach for obtaining a binary-type screening contract is standard (Bolton and Dewatripont, 2005). The fully informative case studied by Guo and Zhang (2012) is an application of 
binary type screening contracts to the context of product line design, and so obtaining the product line in the fully informative case follows the standard approach. In the product line design with quantal consumer preferences, different parameter specifications can alter the approaches used to solve the product line design problem. In this paper, in order to compare with the equilibrium product line in the fully informative case, we make an assumption on the quantal preferences that allows us to use an approach that follows the standard approach for solving typical screening contract problems. Our analysis of the properties of the equilibrium product line is based on the equilibrium obtained by this solving approach.

The quantal preferences significantly change the incentive a product line can provide to consumers, and hence alter several standard results for the screening contract. We find that the difference between the valuation of high preference and low preference towards a type of product plays a crucial role in determining the equilibrium properties that are different from those of conventional screening contracts. For example, even if the low-preference consumers can get a positive surplus in our model by choosing the low-type product (as opposed to zero surplus in any binary type screening contract), the difference between the valuation of high preference and low preference needs to be high enough to retain the result, as does the high preference itself. The quantal feature makes preferences decisive in determining particular properties different from the product line properties in the fully informative case.

When considering the quantal preferences, an equilibrium product line will possess two additional incentives, compared with the fully informative case. It needs to drive consumers to deliberate to find out their true preferences as precisely as possible and to limit the surplus losses for consumers who after deliberation perceive a wrong preference. In the fully informative case by Guo and Zhang (2012), the equilibrium contract only exhibit three incentives. The three incentives are motivating consumers to participate the purchasing activity, motivating consumers to deliberate and consequently find out their true preference, and incentivizing consumers to truthfully determine their preferences.

Besides, due to the presence of quantal preferences, not only more incentives are loaded onto the product line, but also these incentives can be interrelated with each other. We find that the incentive of motivating consumers to truthfully determine their perceived preferences interacts with the incentive of limiting the surplus losses for consumers who perceive a wrong preference after deliberation, and this interaction essentially determines whether the quality of the products, both high and low type, can be larger or smaller than the quality of the same type of product in the fully informative case. The deliberation cost adjusts which incentive is the dominant factor when influencing the quality of the product.

The quantal preferences make both the high type product and low type product more attracting for consumers with low preference and high preference respectively. The high type product, in the product line design with quantal preferences, can attract demand from low preference consumers, which does not happen in the fully informative setting and similar results cannot be obtained in any conventional binary type screening contract. For the product line in the fully informative setting, producing high type product can always get a positive profit, which features conventional binary type screening contract. The quality of the high-type product is always positive and it always brings positive surplus for high-type consumers. Hence, in the fully informative setting, the production of high type product will not be ceased.

However, by considering the quantal feature of preferences, the profit for the high type product can be negative in some situations, in which the production of high type product shall be ceased. For the low type product, in the fully informative setting, if it is not produced, the reason can only be that its quality is negative. But in the product line design with quantal preferences, negative profit or negative surplus for low-preference consumers can arise even if the quality of the low type product is positive, and hence the production of low type product is ceased. Therefore, by considering the quantal feature of preferences, the decision of which type of product shall be produced becomes complicated. It is no longer for sure that if only one type of product can be produced, it will be the high-type product.

In this comprehensive paper, we exhibit several important properties that fundamentally differentiate a screening contract that considers quantal preference from a conventional screening contract, where the agents' hidden information is always clear to the agents themselves. The product line of the fully informative setting by Guo and Zhang (2012) is an example of a conventional screening contract. How quantal preferences affect strategic mechanisms like auctions, moral hazard problems etc., as well as other agency problems (e.g. product line design in our paper), requires research in further depth. The paper also provides a systematic framework for firms to design a product line, e.g. a line of consumer finance products, to protect consumers' interests while securing their own best interests. We expect this paper will provide enough incentives to motivate relevant research in the future.

This paper is organized as follows. In Section 2, we review related literature. In Section 3, we frame the product line design problem by considering the quantal consumer preferences and derive the equilibrium product line. Section 4 analyzes the equilibrium properties of the product line and the intuitions behind these properties. Section 5 summarizes and concludes this paper.

\section{Related Literature}

As documented in Buttler and Loomes (2007), the idea that preferences can be imprecise, or that stated or expressed preferences are inaccurate, can be dated back to Fechner (1860). 
The phenomenon that human beings' perceived preferences may not be their true preferences were formally discovered and stated in the works by Harless and Camerer (1994) and Hey and Orme (1994). The main intent of both papers is to explain the occasional violation of expected utility theory in experiments' results and attempt to generalize expected utility theory. The phenomenon that stated preferences are inaccurate is a critical prerequisite to implementing estimation on the experimental data. For both papers, in order to implement their estimation approaches, they assume the experimental data on stated preferences contain errors. Hence, the stated preferences in the data may not be the subjects' true preferences. In making this assumption, both papers think that intuitively these errors should exist. In Harless and Camerer (1994), they are just regarded as the errors in expressing true preferences. These errors happen with a fixed probability $\varepsilon$ (p. 1260, Harless and Camerer, 1994). There is no detailed explanation or attempt in Harless and Camerer (1994) about the sources of these errors, and why they can be modelled as randomness that happens with a fixed probability. Hey and Orme (1994) provide a more detailed and sensible explanation to support this assumption. They state that these errors can be caused by mistakes, carelessness, slips, inattentiveness, etc. In their work, the errors are modelled as a white noise that follows a normal distribution with zero mean and additive to the values expressed by the preference functionals, because it is "possible the most natural assumption for an economist to make" (p.1301, Hey and Orme, 1994). Even though at the beginning it is assumed that the errors can exist in stated preference data, their results show that the errors do exist in the stated preference data. In essence, the assumption and intuition that the stated or expressed preferences are noisy and imprecise gets support from evidence.

After Harless and Camerer (1994) and Hey and Orme (1994), Loomes and Sugden (1995), based on the work by Becker, DeGroot and Marschak (1963), propose another model that incorporates randomness into an individual's preference. The idea is that for each individual, there is a set of preference functionals, and at each round of decision making, the individual randomly picks up one preference functional as his preference.

In this paper, the distribution that describes the phenomenon that the expressed preferences may not be humans' true preferences is called quantal preferences. The models proposed by Harless and Camerer (1994), Hey and Orme (1994), and Loomes and Sugden (1995) that incorporate random errors into an individual's preference can be regarded as concrete examples that describe quantal preferences. In expectation, a lot of theory, especially in cognitive psychology, can explain the formation of the quantal preferences. However, in this paper, we focus on the impact that quantal preferences bring on contract design in a product line context. For the formation of quantal preferences, we will address this issue in separate researches.

The studies on errorneous stated preferences in experi- ments did not proceed until it was utilized in the research on measuring preference imprecision. In Loomes (2005), he views preference imprecision equivalent to the noises in stated preferences. Even if it is still controversial whether the two concepts can be equivalent, there is no doubt that these two concepts are closely related (Cubitt, Navarro-Martinez and Starmer, 2015). Preference imprecision is often measured by preference interval. The term imprecision interval is proposed by Buttler and Loomes (2007), and from its definition, we can also tell the meaning of preference imprecision:

“...'imprecision interval'...mean the range where an individual finds it hard to say whether any particular value is preferable to or less preferred than the stimulus, and therefore the range where no one value is obviously better or worse than another as an expression of equivalence for the stimulus being evaluated." (pp. 280-281, Buttler and Loomes, 2007) Put simply, preference imprecision can be understood as:

"I'm not sure about my preference." (p.1, Cubitt, NavarroMartinez and Starmer, 2015).

We think that the erroneous stated preferences and preference imprecision are equivalent, and the two concepts can be linked via the law of large numbers, which relationship is manifested in the definition of quantal preferences. The preference imprecision, according to its exhibited features, can be described by a distribution, e.g. the quantal consumer preferences. According to the definition of quantal preferences, the consumer is not sure what exactly his preference is after a cognitive process for inferring the preference, and this uncertainty can be described by certain statistical regularities. The uncertainty implies that it is possible that in the end, some consumers will mistakenly perceive a wrong preference as their own, which they will only realize after consumption.

As we stated earlier, it is hard to believe that anyone who always swings between two alternative preference will finally make a decision. Therefore, the uncertainty resulting from thinking or deliberation will resolve as a natural consequence. According to our quantal preference model, supposing consumers are homogeneous, when uncertainties are resolved, perceived preferences are indeed true preferences for some people, but for others, they will have perceived a wrong preference. For any particular individual, whether his finally perceived preference is his true one or not is random and hard to know. But for a population, how many people can or cannot correctly perceive their preferences can be known according to law of large numbers that transforms an individual's belief about his type into the proportion of how many people can or cannot correctly perceive their preferences. Thus, the stated preferences in data are erroneous.

Therefore, the errors in the stated preference data for a group of people and the imprecision of an individual's perception of his own preferences are essentially coherent from the perspective of quantal preferences. In quantal preferences, the errors in stated preferences and the imprecision of an 
individual's perception on his own preferences are equivalent. Therefore, the empirical findings and experimental results on preference imprecision also provide evidence support to our quantal preference proposition.

In contract theory, the study that is most close to our paper's consideration is Lewis and Sappington (1993). Lewis and Sappington (1993) consider an adverse selection problem where it is possible that the agents may not know the state information, which is continuously distributed rather than discrete. The agent, therefore, does not necessarily possess more private information than what the principal knows. In their model, the agent can realize that they do not know the state information and will truthfully report this fact if it happens in the contract design. This ignorance in state information introduces several special properties in the equilibrium contract, e.g. the pooling equilibrium. In our paper, the quantal property of consumer preferences can also be viewed as a kind of ignorance on state knowledge for consumers (agents). The difference between the problem considered in our paper and the question studied in Lewis and Sappington (1993) is that in our model, consumers who mistakenly perceive a wrong preference after deliberation, i.e. those who are ignorant on state information, do not realize their ignorance until they finish using the product. That is, until the moment when the contract has been executed. Our consideration of the ignorance on state information is from a behavioral perspective. Therefore, the understandings of the contract design in our paper and in Lewis and Sappington (1993) fundamentally differ, as well as the equilibrium contract and its properties and explanations.

In the discipline of cognitive psychology, deliberation, a cognitive activity that can be assorted to thinking, is usually regarded as an information acquisition process (Lachman, Lachman and Butterfield, 1979). ${ }^{1}$ Therefore, it is worthwhile to investigate whether in existing literature of contract theory, there is any related research that considers information acquisition or information management problems. Interestingly, it is another paper by Lewis and Sappington (1997) that is most close to the information acquisition consideration in our paper. In Lewis and Sappington (1997), they consider a moral hazard problem in the context of procurement, where the agent acquires information on the external environment and then chooses an unobservable level of cost-reducing effort. The difference between the information acquisition in our paper and in Lewis and Sappington (1997) is that the information acquisition activity in our paper is founded in the behavioral perspective and the agent acquires information on one's own innate property, the preference. In addition, the information acquisition process cannot be ideal such that the agents can perfectly know their true preferences due to the limited cognitive ability in information processing. In Lewis and Sappington (1997), after the information acquisition, the agent can perfectly know the state information and truthfully report the state information to the principal. Therefore, even

\footnotetext{
${ }^{1}$ There have been several information processing paradigms and theories proposed in the field of cognitive psychology, for example the Newell-Simon model (Lachman, Lachman, and Butterfield, 1979).
}

though both papers consider information acquisition activity in contract design, the modelling and the thinking of agents' behavior are essentially different, which differentiates our paper from Lewis and Sappington (1997).

In terms of inferring consumers' preference information from a line of products, our paper is also related to Guo and Zhang (2012) and Kamenica (2008). Guo and Zhang (2012) study a product line design problem that follows designing a binary-type screening contract. Consumers need to find out their preferences through a costly deliberation activity, and then choose the high-type or low-type product to match their preferences. After deliberation, consumers can accurately find out their own preferences, which is private information for consumers, and not available to the firm. The firm designs a product line to serve consumers by considering this fact. The difference between our paper and Guo and Zhang (2012) is that in our model, consumers cannot accurately find out their own preferences after deliberation, a state described by the quantal preferences. Consumers are not clear about their own preferences, and some of them perceive an incorrect preference when making purchasing decisions. The imperfect preference information is still consumers' private information. Our product line design with quantal preferences is based on the model by Guo and Zhang (2012), which can be viewed as a benchmark such that after a deliberation given any cost, consumers can always correctly perceive their own preferences. Therefore, we also refer to Guo and Zhang's (2012) product line model as the fully informative case. Throughout the paper, we will compare our model with the fully informative case, from the formulation of the product line design problem to the product line's equilibrium properties. Guo and Zhang's (2012) model is an application of the screening contract, and their results inherits the regular results for screening contracts. Therefore, by comparing the fully informative case, we can discover how quantal preferences change the nature of a binary-type screening contract in any aspect.

Kamenica (2008) presents a model also showing that consumers can infer their preference information according to the context of the product line (the prices and qualities of a list of products as in our model). The difference is that in our model, the preference inference process is conducted via deliberation, a cognitive activity. In our model, the firm does not know consumers' preferences, no matter whether they are correct or incorrect after deliberation, and this fact is reflected in the equilibrium product line. The discussion above has shown how this process proceeds. But in Kamenica (2008), the preference inference is conducted via a rational reasoning process. The firm knows all consumers' preferences, while there are uninformed consumers who do not know their own preferences. The variety of the products provided in the product line contains the information about the consumers' preferences. For uninformed consumers, since they are playing equilibrium, they know that the provided product line contains related information about their preferences and the preference information can be obtained by reasoning through the information involved in the product line. 


\section{Product Line Design for Consumers with Quantal Preferences}

There are two types of consumers in our consideration, characterized according to consumers' preferences. Denote $\theta \in\left\{\theta_{l}, \theta_{h}\right\}$, where $\theta_{l}<\theta_{h}$. $\theta$ represents the consumer's valuation towards a type of product. We say the consumer with valuation $\theta_{h}$ the high preference consumer or the consumer's preference is high, and accordingly the consumer with valuation $\theta_{l}$ the low preference consumer or the consumer's preference is low. The prior probability that a consumer is of high preference is $\alpha \in(0,1)$, and accordingly of low preference $1-\alpha \in(0,1)$. By deliberation, consumers perceive their preferences as either $\theta_{l}$ or $\theta_{h}$, which could be opposite to their true preferences because deliberation as a costly cognitive activity can be erroneous. Define $\beta^{h}$ as the probability that a consumer's perceived preference is high after deliberation given that his true preference is high, $\beta^{l}$ as the probability that a consumer's perceived preference is high after deliberation given that his true preference is low, $\beta$ as the probability that a consumer perceives himself having high preference after deliberation. According to the definition, we have $\beta=\alpha \beta^{h}+(1-\alpha) \beta^{l}$, and accordingly $1-\beta=\alpha\left(1-\beta^{h}\right)+(1-\alpha)\left(1-\beta^{l}\right)$. The consumer preference subject to such a probabilistic form is the mathematical expression of the quantal preference.

Following the manner in cognitive psychology that regards cognitive activities as information acquisition process, we regard deliberation, or rigorously in psychological terms, thinking, as an information acquisition activity. By observing a line of products that incentivize consumers to deliberate, the psychological activity is kicked off. Figure 1 exhibits the entire process of the movement of preference perception. One's own preference is his genuine internal property. However, finding out his true preference with respect to the line of products is a costly information acquisition process from the perspective of cognitive psychology, and hence errors may occur due to the consumer's limited cognitive ability. Therefore, consumers' perceived preferences after the costly cognitive process may not be correct, and the statistical regularities of preference perceptions as the outcome of deliberation are defined as the quantal preferences.

$$
\text { prior } \stackrel{\text { I }}{\rightarrow} \text { posterior } \stackrel{\text { II }}{\rightarrow} \text { perceived preference } \stackrel{\text { III }}{\rightarrow} \text { true preference }
$$

Figure 1: The movement of preference perception, where I: deliberation, II: uncertainty resolved, and III: choose and consume a product.

The movement of preference perception described in Figure 1 is illustrated as follows. Before deliberation begins, consumers hold a prior belief on their preferences, i.e. $(\alpha, 1-\alpha)$. After deliberation, the acquired information on preferences updates the belief and a posterior belief is obtained, i.e. $\left(\beta^{h}, 1-\beta^{h}\right)$ and $\left(1-\beta^{l}, \beta^{l}\right)$. Uncertainty on preferences is finally resolved and every consumer can perceive a deterministic preference, which can be either their own or an incorrect one. Each consumer makes a purchasing decision based on this perceived preference, and only after consuming the product, consumers can know their true preferences. ${ }^{2}$

Intuitively, the smaller the deliberation cost is, the easier people can do it, and the more accurately their quantal preferences can reflect their true preferences. In the extreme case of free deliberation, everyone can obtain his or her true preference correctly, which is the situation considered in Guo and Zhang (2012). A sensible quantal preference is expected to possess such features. These features are consistent with our understanding of quantal preferences and deliberation as a cognitive activity. Denote the deliberation cost by $c$. In the following, for quantal preferences, we make the following assumption throughout the paper:

Assumption 1: For quantal preferences, $\frac{\partial \beta^{h}}{\partial c}<0, \frac{\partial \beta^{l}}{\partial c}>0$, $\lim _{c \rightarrow 0} \beta^{h}=1$ and $\lim _{c \rightarrow 0} \beta^{l}=0$.

Assumption 1 implies that the designed product line not only motivates consumers to deliberate, but also drives consumers to accurately find out their true preferences as much as possible. In the fully informative case, the product line can only motivate consumers to deliberate except incentivizing their participation and truthfully telling their preferences (Guo and Zhang, 2012). Quantal preferences make it possible that the product line affords more incentives.

In the following part of the paper, from the construction of the product line design problem to the analysis of equilibrium, we will study the general features and properties for the product line brought by the quantal consumer preferences, no matter how these preferences are obtained, and according to any theory.

Consider a unit mass of consumers. The product line the firm intends to design contains two types of product, the high-type product and the low-type product. The product line includes both the quality and price of the two types of products, i.e., $\left(p_{h}, q_{h}\right)$ and $\left(p_{l}, q_{l}\right)$. The consumers' preferences for the line of products can be either high or low. The firm first designs the product line, and then the consumers buy the products that are consistent with their preferences. The process is known by both the firm and consumers.

According to the law of large numbers, the posterior belief

\footnotetext{
${ }^{2}$ In the following, by analyzing the product line, we will present that the equilibrium product line exhibits such properties: when the deliberation cost is small, consumers are willing to deliberate and they use the perceived preferences for making decisions; when the deliberation cost is large, although consumers deliberate, they use the expected preferences based on the posterior belief, i.e. $\frac{\alpha \beta^{h} \theta_{h}+(1-\alpha) \beta^{l} \theta_{l}}{\beta}$ and $\frac{\alpha\left(1-\beta^{h}\right) \theta_{h}+(1-\alpha)\left(1-\beta^{l}\right) \theta_{l}}{1-\beta}$, to make decisions rather than the perceived preferences, which are not as reliable as the former due to the low quality of the posterior beliefs by the large deliberation cost; when the deliberation cost is too high for consumers to deliberate, then consumers will make decisions according to expected preference $\theta_{m}$.
} 
$\beta^{h}$ or $\beta^{l}$ also represents the proportion of people who correctly or mistakenly perceive their types within a population of preference type $\theta_{h}$ or $\theta_{l}$. If a firm designs a product line ignoring the quantal property of consumer preferences, the consumers whose perceived preference is opposite to his true preference will experience a wasteful purchase. Consider the benchmark model (the fully informative case) exhibited by Guo and Zhang (2012). For high preference consumers who regard their preferences as low, their surplus is smaller than those who correctly perceive their preference as high, while his surplus is still positive because the low-type product is incentivecompatible for low preference consumers and the valuation of high preference is greater than the valuation of low preference. However, low preference consumers who regard their type as high and buy the high type product, may find the product too expensive so that ex post they find that their surplus is negative.

How, then, can we design a product line where consumers' preferences are quantal, or in a binary type context, some consumers may perceive opposite type of themselves? First of all, we want to ask, whether it is possible for all consumers to get a non-negative surplus even if ex post they find that the purchased product is not consistent with their true preferences? The answer is no. A product line designed following the screening contract paradigm and making all consumers get a non-negative surplus in this case means all participation constraints and incentive compatibility constraints are binding and the prices and qualities are determined by these binding constraints. It is irrelevant to the firm's profit maximization objective. Therefore, it is not desirable for a firm to design such a product line. What the firm can do at best is to limit the surplus losses for those consumers who choose a product that is inconsistent with their true preferences.

The heart of any contract design lies in its incentive compatibility conditions. It is also a natural requirement in our product line design problem. A truth-telling contract not only makes sure an individual does not regret his ex ante truth-telling decisions (ex ante-ex post consistency), but also makes sure the population of a given type of consumers does not regret their choices as well. In the product line design problem, the ex ante-ex post consistency is held when consumers clearly know their preferences after deliberation as reflected in the fully informative case (Guo and Zhang, 2012). However, if the preference information is uncertain for consumers after deliberation, as we just discussed, it is hard to design a contract where the consistency can be perfectly held. In this paper, due to the concern that the presence of quantal preferences may violate the expected utility theory as shown by experimental evidences such as Harless and Camerer (1994) and Hey and Orme (1994), we take the perspective of designing the product line by motivating the population of a given type of consumers to truth-tell rather than by motivating an individual.

Recall from last section that after deliberation, the share of consumers who perceive themselves having high preference is $\beta$, and accordingly $1-\beta$ consumers perceive themsleves having low preference. In a truth-telling contract, the firm can expect that only consumers who think they are of high preference pays $p_{h}$ to buy the product with quality $q_{h}$, while consumers who think their preferences are low pays $p_{l}$ to buy the product with quality $q_{l}$. Therefore, the firm's objective is to design an appropriate product line $\left(\left(p_{l}, q_{l}\right),\left(p_{h}, q_{h}\right)\right)$ to maximize the aggregate profit $\beta\left(p_{h}-\frac{q_{h}^{2}}{2}\right)+(1-\beta)\left(p_{l}-\frac{q_{l}^{2}}{2}\right)$.

The consumers make their purchasing decisions based on their perceived preferences, which are hidden from the firm. However, both firms and consumers know the statistic $\beta, \beta^{h}$ and $\beta^{l}$. As a contract targeting the population of a given type of consumers, it is the solution of the following truth-telling mechanism:

$$
\max _{p_{h}, p_{l}, q_{h}, q_{l}} \beta\left(p_{h}-\frac{q_{h}^{2}}{2}\right)+(1-\beta)\left(p_{l}-\frac{q_{l}^{2}}{2}\right)
$$

s.t.

$$
\begin{gathered}
\beta^{h}\left(\theta_{h} q_{h}-p_{h}\right)+\left(1-\beta^{h}\right)\left(\theta_{h} q_{l}-p_{l}\right) \geq 0 \quad\left(P C^{h}\right) \\
\beta^{l}\left(\theta_{l} q_{h}-p_{h}\right)+\left(1-\beta^{l}\right)\left(\theta_{l} q_{l}-p_{l}\right) \geq 0 \quad\left(P C^{l}\right) \\
\beta^{h}\left(\theta_{h} q_{h}-p_{h}\right)+\left(1-\beta^{h}\right)\left(\theta_{h} q_{l}-p_{l}\right) \geq \beta^{h}\left(\theta_{h} q_{l}-p_{l}\right)+\left(1-\beta^{h}\right)\left(\theta_{h} q_{h}-p_{h}\right) \\
\left(I C^{h}\right) \\
\beta^{l}\left(\theta_{l} q_{h}-p_{h}\right)+\left(1-\beta^{l}\right)\left(\theta_{l} q_{l}-p_{l}\right) \geq \beta^{l}\left(\theta_{l} q_{l}-p_{l}\right)+\left(1-\beta^{l}\right)\left(\theta_{l} q_{h}-p_{h}\right)
\end{gathered}
$$$$
\left(I C^{l}\right)
$$$$
U\left(\beta^{h}, \beta^{l}\right)-c \geq \max \left(\theta_{m} q_{h}-p_{h}, \theta_{m} q_{l}-p_{l}, 0\right) \quad\left(I C^{d}\right)
$$

where $U\left(\beta^{h}, \beta^{l}\right)=\alpha \beta^{h}\left(\theta_{h} q_{h}-p_{h}\right)+\alpha\left(1-\beta^{h}\right)\left(\theta_{h} q_{l}-\right.$ $\left.p_{l}\right)+(1-\alpha) \beta^{l}\left(\theta_{l} q_{h}-p_{h}\right)+(1-\alpha)\left(1-\beta^{l}\right)\left(\theta_{l} q_{l}-p_{l}\right)$ and $c$ is the deliberation cost. Consider the participation constraints that target the population of consumers with a given type of preference. These constraints put a restriction on the potential surplus losses for the consumer who perceives a wrong preference of themselves after deliberation. Take $P C^{l}$ as an example. In the fully informative case where consumers can accurately find out their true preferences by deliberation, as long as $\theta_{l} q_{l}-p_{l} \geq 0$, the low preference consumers are willing to participate the purchasing activity. But now, the participation constraints become more complicated. For the convenience of illustration, it can be expressed by $\theta_{l} q_{l}-p_{l} \geq-\frac{\beta^{l}}{1-\beta^{l}}\left(\theta_{l} q_{h}-p_{h}\right)$.

If the surplus for low preference consumers who mistakenly perceive themselves having high preference is negative, i.e. $\theta_{l} q_{h}-p_{h}<0$, the requirement that motivates low preference consumers who correctly find out their types to participate the purchasing activity becomes stricter. $\theta_{l} q_{l}-p_{l}$ has to be greater than a positive value $-\frac{\beta^{l}}{1-\beta^{l}}\left(\theta_{l} q_{h}-p_{h}\right)$ so that low preference consumers who correctly find out their types are willing to participate the purchasing activity. The same rationale also applies to high preference consumers. 
From another perspective, we can transform $P C^{l}$ by $\theta_{l} q_{h}-p_{h} \geq-\frac{1-\beta^{l}}{\beta^{l}}\left(\theta_{l} q_{l}-p_{l}\right)$. It indicates that in the product line design that considers quantal consumer preferences, limiting the surplus losses for low preference consumers who mistakenly perceive themselves having high preference is taken into consideration by the firm in the product line design. The same rationale also applies to high preference consumers.

Note that in the fully informative case, the possibility that some consumers may not correctly find out their true preference types after deliberation is excluded in the product line design. Hence, these consumers' surplus losses are not counted in the fully informative case, and consumers who correctly perceive their preferences have no motivation and do not have any extra surpluses to compensate for the consumers who perceive a wrong preference. Thus, in the fully informative case, the surplus losses would not be compensated for from the designed product line. As we will exhibit below, to motivate all consumers to participate the purchasing activity by deliberation, a firm will sacrifice some of its profits relative to what it could have obtained in the fully informative case to reimburse consumers. For consumers who mistakenly perceive their preferences, their surplus losses can be limited from this reimbursement. Besides, for those who correctly perceive their types, they can obtain extra surplus from the surplus losses of those who perceive a wrong preference after deliberation, which is a potential source to compensate the surplus losses for those perceiving a wrong preference. How to transfer the extra surplus to compensate consumers experiencing surplus losses is out of scope of this paper.

Consider the incentive compatibility constraints that target the population of consumers with a given type of preference, the $I C^{h}$ and $I C^{l}$. Each constraint indicates for a population of high preference or low preference consumers, it is in their interests for the group of people to truth-tell. At the individual level, a consumer makes his truth-telling decisions based on his perceived preference. Therefore, to understand the population-oriented incentive compatibility constraints' impact on each individual, it is desirable to reformulate them in accordance to incentive-compatibility constraints for the individual. The $I C^{h}$ and $I C^{l}$ can be rewritten into the following expressions by collecting terms:

$$
\begin{gathered}
\left(2 \beta^{h}-1\right)\left(\theta_{h} q_{h}-p_{h}\right) \geq\left(2 \beta^{h}-1\right)\left(\theta_{h} q_{l}-p_{l}\right) \quad\left(I C^{h^{\prime}}\right) \\
\left(1-2 \beta^{l}\right)\left(\theta_{l} q_{l}-p_{l}\right) \geq\left(1-2 \beta^{l}\right)\left(\theta_{l} q_{h}-p_{h}\right) \quad\left(I C^{l^{\prime}}\right)
\end{gathered}
$$

$I C^{h^{\prime}}$ and $I C^{l^{\prime}}$ show that how the truth-telling constraints adapt to individuals depends on $\beta^{h}$ and $\beta^{l}$. If $\beta^{h}>\frac{1}{2}$ where high preference people who correctly perceive their preferences make up the majority, then the incentive-compatibility constraint towards the high preference population reduces to $\theta_{h} q_{h}-p_{h} \geq \theta_{h} q_{l}-p_{l}$, which motivates high preference consumers who correctly perceive their preferences to choose the high-type product. Suppose $\beta^{l}<\frac{1}{2}$ and hence $I C^{l^{\prime}}$ reduces to $\theta_{l} q_{l}-p_{l} \geq \theta_{l} q_{h}-p_{h}$. According to this reduced constraint, for the high preference people who mistakenly perceive their preferences as low, they are also truth-telling by choosing the low-type product. However, ex post, these high preference people regret their choices because according to the reduced $I C^{h^{\prime}}$ constraint $\theta_{h} q_{h}-p_{h} \geq \theta_{h} q_{l}-p_{l}$, their surpluses are smaller than what they could have obtained if they had perceived their preferences correctly. Therefore, given that $\beta^{h}>\frac{1}{2}$ and $\beta^{l}<\frac{1}{2}$, it is both $I C^{h^{\prime}}$ and $I C^{l^{\prime}}$ that motivates all high preference people, as a population, to truth-tell their perceived preferences. For high preference consumers who perceive a wrong preference after deliberation, ex ante-ex post consistency cannot be held due to their unanticipated surplus losses. The same conclusion also applies to the low preference consumers.

Note that $\beta^{l}<\frac{1}{2}$ indicates that for low preference consumers, people who correctly perceive their preferences make up the majority. It makes sense that after deliberation, the majority of consumers of a given type can correctly find out their types. Hence in the following of the paper, we will focus on analyzing the case where $\beta^{h}>\frac{1}{2}$ and $\beta^{l}<\frac{1}{2}$.

Here, by only assuming $\beta^{h}>\frac{1}{2}$ and $\beta^{l}<\frac{1}{2}$, we can make a comparison of a firm's profit between our model and the fully informative case. Given that $\beta^{h}>\frac{1}{2}$ and $\beta^{l}<\frac{1}{2}$, according to $P C^{h}$ and $P C^{l}$, the designed product line will make consumers who correctly find out their types get more surplus after deliberation than in the fully informative case as a necessary step to ensure the consumer population of each type is willing to participate the purchasing activity. As we stated after formulating the contract design problem, the $P C^{h}$ and $P C^{l}$ are more complicated than the participation constraints in the benchmark case. $I C^{h}$ and $I C^{l}$ are of the same expression as in the benchmark case given that $\beta^{h}>\frac{1}{2}$ and $\beta^{l}<\frac{1}{2}$. The deliberation motivation constraint $I C^{d}$ is tighter than its counterpart in the benchmark case. Therefore, the solution set described by the constraints in the product line design problem that considers quantal consumer preferences cannot be broader than the solution set in the fully informative case. And therefore, the profit for each segment of consumers $p_{h}-\frac{q_{h}^{2}}{2}$ and $p_{l}-\frac{q_{l}^{2}}{2}$, as well as the aggregate profit even if there is a reshuffling of consumers after deliberation, cannot be larger than the corresponding profit in the benchmark case respectively. The reduced profit relative to its counterpart in the fully informative case is transferred to consumers, which is used to limit the surplus losses for consumers who perceive a wrong type after deliberation and motivate each population of consumers to participate the purchasing activity.

Therefore, in the product line design with quantal consumer preferences, the limitation of surplus losses is contributed by the firm sacrificing some of its profit. For the extra surplus, which is the additional surplus obtained for each type of consumers who correctly find out their types relative to their surplus in the fully informative case, it is obtained from the surplus losses of the consumers of the same type but perceive an incorrect preference after deliberation, and from the firm's sacrifice of its profit. In general, the product line design with 
quantal consumer preferences is consumer-friendly. The firm will subsidize consumers to participate the purchasing activity.

Only when the deliberation cost $c$ equals zero is our model the same as the fully informative case where the deliberation cost $c$ equals zero. In this situation, the firm's objective function will be the same as the one in the fully informative case. In this situation, $\beta^{h}$ equals one and $\beta^{l}$ equals zero, and all constraints become most slack, i.e., the solution set determined by the constraints becomes largest. Therefore, at $c$ equal zero, the profit for selling each type of product in our model reaches its largest respectively. Hence, it is in the firm's interest to help consumers enhance their ability to deliberate to find out their true preferences. Although the same result is also obtained in the fully informative case, the intuitions come from different sources. The intuition obtained in our model is driven by a behavioral consideration that how deliberation cost affects consumers' perception towards their own preferences. In Guo and Zhang (2012), it is caused by the mechanism that deliberation becomes less costly and hence the firm can compensate less for consumers' deliberation activities.

Typically, for a conventional binary type screening contract, e.g., the benchmark case of Guo and Zhang (2012), $P C^{l}$ and $I C^{h}$ implies $P C^{h}$ (Bolton and Dewatripont, 2005). However, by considering the quantal property of consumer preferences, this regularity would not be held unless certain conditions are satisfied. We find that

Proposition 1: Given that $\beta^{h}>\frac{1}{2}$ and $\beta^{l}<\frac{1}{2}, P C^{l}$ and $I C^{h}$ imply $P C^{h}$ if and only if $1-\beta^{l^{2}} \geq \beta^{h}$.

The necessary and sufficient condition in Proposition 1 indicates that unless the proportion of consumers of low preference who correctly perceive their preferences is larger than the proportion of consumers of high preference who correctly perceive their preferences, $P C^{l}$ and $I C^{h}$ do not imply $P C^{h}$ and hence we cannot apply the conventional approach to simplify the contract design problem.

The condition $1-\beta^{l} \geq \beta^{h}$ means the proportion of low preference consumers who correctly find out their preferences after deliberation is greater than the proportion of high preference consumers who correctly find out their preferences after deliberation. Researches in economics and psychology have shown that the accuracy of decision is related with the complexity of the problem. In complex problems, agents need more time to get precise information to make decisions and hence mistakes are more likely to happen (Fudenberg, Strack and Strzalecki, 2018). Therefore, given that incentive compatibility constraints are held, which indicates that the choice decision is consistent with the agents' perceived preferences, when consumers face a line of products that include a product that is complex in nature, they will spend more time to deliberate and hence are more likely to perceive a wrong preference. High type product is usually more complex in terms of their quality, design, etc., than low type product. Therefore the wrong choice is more likely to make when evaluating high type product than low type product. Financial product like mutual fund can be viewed as a high type product, while savings can be viewed as a low type product. Apparently, mutual fund is more complicated and difficult to understand than savings for consumers, and hence consumers have to spend more time to evaluate a mutual fund than savings. Therefore, mistakes are more likely to happen when facing such a line of products. Because savings are easier to evaluate, there are more consumers who generically prefer savings choosing savings than consumers who generically prefer mutual fund choosing a mutual fund. Therefore, the result $1-\beta^{l} \geq \beta^{h}$ happens.

Assumption 2: Assume $\beta^{h}>\frac{1}{2}$ and $\beta^{l}<\frac{1}{2}$, and $1-\beta^{l} \geq \beta^{h}$.

In the following, we will focus on the case where Assumption 2 is held. The product line design problem subject to Assumption 2 parallels the fully informative case as studied by Guo and Zhang (2012) in both the formulation of the problem and the approach for solving the problem. Therefore, the comparison of equilibrium product line between our model and the fully informative case is feasible and meaningful. For cases where Assumption 2 is not held, we will discuss them in Section 5.

Given that Assumption 2 is held and hence $P C^{l}$ and $I C^{h}$ imply $P C^{h}, P C^{l}$ should be binding. Otherwise the firm can increase the price of both high type and low type product to increase its profit. Consumers can make purchasing decisions either by deliberation or based on the expected preference $\theta_{m}$ where no deliberation is made. Therefore, to motivate consumers to deliberate, the firm has to pay enough cost incurred by consumer deliberation. The incentive to motivate consumers to deliberate rather than just relying on expected preference is constructed via the constraint $I C^{d}$. In our model, by considering the impact of deliberation cost on quantal preferences as presented in Assumption 1, the payment for deliberation cost not only motivates consumers to deliberate, as in the fully informative case (Guo and Zhang, 2012), but also drives the consumers to find out their true preferences as accurately as possible via the deliberation process.

In addition, in the process of deriving the equilibrium product line under Assumption 2, we find that, the incentive compatibility constraint $I C^{d}$ can be decomposed into two separate incentive compatibility constraints $I C_{h}^{d}$ and $I C_{l}^{d}$, which motivate consumers who hold the same perceived preferences to truth-tell their perceived preferences after deliberation. Besides, given $I C^{h}, I C_{h}^{d}$ implies $I C^{l}$, and given $I C^{l}, I C_{l}^{d}$ implies $I C^{h}$.

$p_{h}-p_{l} \geq \frac{\alpha\left(1-\beta^{h}\right) \theta_{h}+(1-\alpha)\left(1-\beta^{l}\right) \theta_{l}}{1-\beta}\left(q_{h}-q_{l}\right)+\frac{c}{1-\beta}$ $\left(I C_{h}^{d}\right)$ 


$$
p_{h}-p_{l} \leq \frac{\alpha \beta^{h} \theta_{h}+(1-\alpha) \beta^{l} \theta_{l}}{\beta}\left(q_{h}-q_{l}\right)-\frac{c}{\beta} \quad\left(I C_{l}^{d}\right)
$$

In the fully informative case of Guo and Zhang (2012), such results do not exist. In the fully informative case, consumers always obtain the true preference after deliberation, and they honestly reveal the obtained true preferences according to the incentive compatibility constraints derived from the constraint that motivate consumers to deliberate. The derived constraints are obtained without any assumption and they are independent from each other. Each incentive compatibility constraint for consumers to truth-tell after deliberation implies the corresponding incentive compatibility constraint for consumers to truth-tell supposing they knew their true preferences with no deliberation, whose formulation follow the reduced $I C^{h^{\prime}}$ and $I C^{l^{\prime}}$ constraints in our quantal preference model given Assumption 2 held. Therefore, in the fully informative case, the consideration of incentivizing one type of consumers to truth-tell is independent from the consideration of incentivizing the other type of consumers to truth-tell in the product line design.

However, in the product line design with quantal preferences, constraint $I C_{h}^{d}$ shows that given high preference consumers truth-telling supposing they knew their preferences, incentivizing consumers whose perceived preference is low after deliberation to truth-tell implies incentivizing low preference consumers to truth-tell supposing they knew their preferences. Similarly, constraint $I C_{l}^{d}$ shows that given low preference consumers truth-telling supposing they knew their preferences, incentivizing consumers whose perceived preference is high after deliberation to truth-tell implies incentivizing high preference consumers to truth-tell supposing they knew their preferences. Therefore, due to quantal preferences, the reduced $I C^{h^{\prime}}$ constraint is related with the reduced $I C^{l^{\prime}}$ constraint subject to Assumption 2, which indicates that the consideration of incentivizing one type of consumers to truthtell depends on the consideration of incentivizing the other type of consumers to truth-tell in the product line design. Therefore,

Corollary 1: Due to the presence of quantal preferences, under Assumption 2, the $I C^{h^{\prime}}$ constraint in its reduced form is related with the $I C^{l^{\prime}}$ constraint in its reduced form.

The equilibrium prices for high type and low type products are

$$
\begin{gathered}
p_{h}=A_{h} q_{h}+B_{h} q_{l}-\frac{\left(1-\beta^{l}\right) c}{\beta} \\
p_{l}=A_{l} q_{h}+B_{l} q_{l}+\frac{\beta^{l} c}{\beta}
\end{gathered}
$$

where $A_{h}=\frac{\alpha \beta^{h}\left(1-\beta^{l}\right)}{\beta} \theta_{h}+\beta^{l}\left[1+\frac{(1-\alpha)\left(1-\beta^{l}\right)}{\beta}\right] \theta_{l}$, $B_{h}=-\left(\theta_{h}-\theta_{l}\right) \frac{\alpha \beta^{h}\left(1-\beta^{l}\right)}{\beta}, A_{l}=-\left(\theta_{h}-\theta_{l}\right) \frac{\alpha \beta^{h} \beta^{l}}{\beta}$, and
$B_{l}=\frac{\alpha \beta^{h} \beta^{l}}{\beta} \theta_{h}+\left(1-\frac{\alpha \beta^{h} \beta^{l}}{\beta}\right) \theta_{l}$. The derivation of $p^{h}$ and $p^{l}$ are documented in Appendix B.

Equation 1 implies that in this product line design problem, like the benchmark case, the low-type product can impact high type product's pricing. However, equation 2 implies that, unlike the benchmark case, by considering the quantal preferences, at equilibrium the high-type product can also impact low type product's pricing. This is because due to the noises in the deliberation process, some high preference consumers mistakenly perceive themselves as low preference consumers, and these consumers form part of the demand of the low-type product. When designing the low-type product, these consumers' purchasing activity is also considered. The negativity of $A_{l}$ reflects the firm's consideration of limiting the surplus losses for consumers who perceive a wrong preference after deliberation. Therefore, the type of product they could have bought, the high type product, has to get involved in the low type product's pricing.

We find that there exists a threshold of the deliberation cost $\bar{c}$ such that for $c \leq \bar{c}$, the equilibrium product qualities are the interior solutions:

$$
\begin{gathered}
q_{h}=\left(\frac{\alpha \beta^{h}}{\beta}-\frac{\alpha \beta^{h} \beta^{l}}{\beta^{2}}\right) \theta_{h}+\left[\frac{\alpha \beta^{h} \beta^{l}}{\beta^{2}}+\frac{(1-\alpha) \beta^{l}}{\beta}\right] \theta_{l} \\
q_{l}=\frac{\alpha \beta^{h}\left(\beta^{l}-\beta\right)}{\beta(1-\beta)} \theta_{h}+\left[1-\frac{\alpha \beta^{h}\left(\beta^{l}-\beta\right)}{\beta(1-\beta)}\right] \theta_{l}
\end{gathered}
$$

For $c>\bar{c}$, the equilibrium product qualities are the boundary solutions: $q_{h}=\theta_{l}+\frac{(1-\beta) c}{\alpha\left(\beta^{h}-\beta\right) \theta_{h}+(1-\alpha)\left(\beta^{l}-\beta\right) \theta_{l}}$, and $q_{l}=\theta_{l}-\frac{\beta c}{\alpha\left(\beta^{h}-\beta\right) \theta_{h}+(1-\alpha)\left(\beta^{l}-\beta\right) \theta_{l}}$, where $\bar{c}=\frac{\alpha \beta^{h}\left(\beta-\beta^{l}\right)}{\beta^{2}(1-\beta)}\left(\theta_{h}-\theta_{l}\right)\left[\alpha\left(\beta^{h}-\beta\right) \theta_{h}+(1-\alpha)\left(\beta^{l}-\beta\right) \theta_{l}\right]$. The derivation of $q_{h}$ and $q_{l}$ are documented in Appendix B.

Finally, for $c<\bar{c}, \Delta q=\phi\left(\theta_{h}-\theta_{l}\right)$, where $\phi=\frac{\alpha \beta^{h}}{\beta}\left(1-\frac{\beta^{l}}{\beta}-\frac{\beta^{l}-\beta}{1-\beta}\right)$ and it is positive. The proof is documented in Appendix $\mathrm{C}$ For $c \geq \bar{c}$, $\Delta q=\frac{c}{\alpha\left(\beta^{h}-\beta\right) \theta_{h}+(1-\alpha)\left(\beta^{l}-\beta\right) \theta_{l}}$. It can be either positive or negative while not equals zero and the proof can refer to Appendix E.

\section{Analysis of Equilibrium}

First, we exhibit properties of the equilibrium that are same as in the fully informative case. Introducing quantal feature does not change these properties.

Proposition 2: In the product line design with quantal consumer preferences, only separating equilibrium exists. For any deliberation $\operatorname{cost} c$, the surplus is positive for consumers whose true preference is high buying high-type product, i.e. $\theta_{h} q_{h}-p_{h}>0$, and the quality of low-type product is below 
the efficient level, i.e. $q_{l}<\theta_{l}$.

The fact that only separating equilibrium exists shows that the product line designed by considering the quantal preferences essentially still follows the purpose of screening contracts. That is, to sort out different types of agents (consumers in our context). However, in our model, the screening task becomes more challenging: besides incentivizing consumers to truth-tell their perceived preferences, firms have to drive consumers to correctly identify their true preferences by the costly deliberation process as much as possible. The instruments to implement the incentive to sort out different types of consumers and to drive consumers to correctly identify their types are same, which are the designed product line. Therefore, the same instrument is loaded with more incentives. Hence the properties of the product line designed subject to the quantal preferences are expected to be distinct from the properties of the product line in the fully informative case in many aspects. In the following, we exhibit some specific properties that are caused by the quantal preferences and do not arise in the fully informative case.

Lemma 1: The threshold $\bar{c}$ and its counterpart $\bar{c}^{f}$ in the fully informative case satisfy the following relationship: $\bar{c}<\frac{\left(\theta_{h}-\theta_{l}\right)\left[\alpha\left(\beta^{h}-\beta\right) \theta_{h}+(1-\alpha)\left(\beta^{l}-\beta\right) \theta_{l}\right]}{1-\beta}<\bar{c}^{f}$. In the fully informative case, $\bar{c}^{f}$ equals $\alpha\left(\theta_{h}-\theta_{l}\right)^{2}$ (Guo and Zhang, 2012).

Lemma 1 indicates that the fully informative case allows broader range of deliberation cost for obtaining interior solutions. As we analyzed in the last section, the range of interior solutions represented by the constraints in the product line design is narrower than in the fully informative case, and the profit of selling each type of product cannot be larger than in the fully informative case respectively. The interior solution completely reflects the firm's profit maximization purpose. For the boundary solutions, the firm is constrained by the fact that consumers whose perceived preferences are high are indifferent between the two types of products after deliberation, and their profit maximization consideration cannot be as free as in the case of interior solutions. Lemma 1 shows that, due to the quantal preferences, the requirement to obtain an interior solution becomes stricter. As long as deliberation is costly, there will always exist some consumers who mistakenly perceive an incorrect preference, and the firm has to motivate all consumers to truth-tell their perceived preferences by considering their purchasing decisions given the deliberation cost. Therefore, in our model, the more complicated consumer composition makes the firm's profit maximization action more restrained than in the fully informative case.

Proposition 3: For $c \leq \bar{c}, q_{h}>0$ if and only if $\frac{\alpha \beta^{h} \theta_{h}+(1-\alpha) \beta^{l} \theta_{l}}{\theta_{h}-\theta_{l}}>\frac{\alpha \beta^{h} \beta^{l}}{\beta}$. For $c>\bar{c}, q_{h}>0$ if and only if $\frac{\alpha \beta^{h} \theta_{h}+(1-\alpha) \beta^{l} \theta_{l}}{\beta}>\alpha \theta_{h}+(1-\alpha) \theta_{l}$ or $\frac{\alpha \beta^{h} \theta_{h}+(1-\alpha) \beta^{l} \theta_{l}}{\beta}<-\frac{(1-\beta) c}{\beta \theta_{l}}+\alpha \theta_{h}+(1-\alpha) \theta_{l}$.
Proposition 4: For deliberation cost $c$ below $\frac{\left(\theta_{h}-\theta_{l}\right)\left[\alpha\left(\beta^{h}-\beta\right) \theta_{h}+(1-\alpha)\left(\beta^{l}-\beta\right) \theta_{l}\right]}{1-\beta}$, the quality of the high-type product is below the efficient level, i.e. $q_{h}<\theta_{h}$. For deliberation cost satisfying $\frac{\left(\theta_{h}-\theta_{l}\right)\left[\alpha\left(\beta^{h}-\beta\right) \theta_{h}+(1-\alpha)\left(\beta^{l}-\beta\right) \theta_{l}\right]}{1-\beta} \leq$ $c<\bar{c}^{f}$, the quality of the high-type product is beyond the efficient level, i.e. $q_{h} \geq \theta_{h}$. For deliberation cost greater than or equal to $\bar{c}^{f}$, the quality of high-type product is greater than its counterpart in the fully informative case $q_{h}^{f}$, which is a boundary solution. In the fully informative case, the $q_{h}^{f}$ equals $\theta_{l}+\frac{c}{\alpha\left(\theta_{h}-\theta_{l}\right)}$ and it satisfies $q_{h}^{f} \geq \theta_{h}$ (Guo and Zhang, 2012).

Compared with the fully informative case, Proposition 4 shows that quantal preference makes the quality of the high-type product either smaller or larger than in the fully informative case. In a standard screening contract, the hightype product is always designed with the efficient quality when incomplete information on consumers' preferences is present for the firm. An aim of keeping the high-type product with efficient quality is to prevent low preference consumers from mimicing the high preference consumers. However, as we see, when the deliberation cost is below $\frac{\left(\theta_{h}-\theta_{l}\right)\left[\alpha\left(\beta^{h}-\beta\right) \theta_{h}+(1-\alpha)\left(\beta^{l}-\beta\right) \theta_{l}\right]}{1-\beta}$, the quality of the high-type product is below the efficient level. When we formulate the contract design problem, we have found that the firm will limit the surplus losses for consumers who perceive a wrong type in its designed product line. Therefore, the quality of a designed product not only expresses the firm's intent to make it consistent with consumers' perceived preferences, but also limit the surplus losses for those consumers who ultimately buy the product due to a wrong deliberation result.

Intuitively, the two forces that affect the product quality play in opposite directions: the first is to perform the incentive compatibility function, i.e., preventing the consumers whose perceived preference is low from mimicing the consumers whose perceived preference is high, that the high-type product should keep its quality high as much as possible; the second is to limit the surplus losses for consumers whose true preference is low from buying the high-type product, that the quality of the high type product has to be low to limit its price and make buying it incur surplus losses as few as possible retrospectively. The result of Proposition 4 shows that, when deliberation cost is below $\frac{\left(\theta_{h}-\theta_{l}\right)\left[\alpha\left(\beta^{h}-\beta\right) \theta_{h}+(1-\alpha)\left(\beta^{l}-\beta\right) \theta_{l}\right]}{1-\beta}$, the concern of limiting the surplus losses for low preference consumers whose perceived preference is high dominates the concern of incentive compatibility, and hence the quality of high-type product is below efficient level. When the deliberation cost is greater than or equal to $\frac{\left(\theta_{h}-\theta_{l}\right)\left[\alpha\left(\beta^{h}-\beta\right) \theta_{h}+(1-\alpha)\left(\beta^{l}-\beta\right) \theta_{l}\right]}{1-\beta}$, the concern of incentive compatibility dominates the concern of limiting the surplus losses of the low preference consumers who mistakenly perceive a wrong type after deliberation and hence $q_{h}$ is greater than or equal to $\theta_{h}$.

Corollary 2: For deliberation cost below $\bar{c}$, the quality of high type product is positive if and only if 
$\frac{\theta_{l}}{\theta_{h}}>-\frac{\alpha \beta^{h}\left(1-\frac{\beta^{l}}{\beta}\right)}{\beta^{l}\left[\frac{\alpha \beta^{h}}{\beta}+(1-\alpha)\right]}$. For deliberation cost greater than or equal to $\bar{c}$, the quality of high type product is always positive.

An important insight Corollary 2 provides is that high type product is possible to have a negative quality and hence not to produce, which is contrary to the conclusion in the fully informative case of Guo and Zhang (2012) or any standard binary-type screening contract, where it is only the low type product that is possible not to produce and high type product always has a positive quality (Bolton and Dewatripont, 2005; Guo and Zhang, 2012). This is a significant difference for binary-type screening contract by considering the quantal preferences from the conventional binary-type screening contract.

Lemma 2: If $\alpha>1-\frac{1}{\frac{\beta^{h}\left(\beta-\beta^{l}\right)}{\beta(1-\beta)}}, \bar{c}<\frac{1}{\beta} \frac{\alpha}{1-\alpha}\left(\theta_{h}-\theta_{l}\left[\alpha\left(\beta^{h}-\right.\right.\right.$ $\left.\beta) \theta_{h}+(1-\alpha)\left(\beta^{l}-\beta\right) \theta_{l}\right]<\bar{c}^{f}$.

Proposition 5: Define $q_{l}^{f}$ as the quality of low-type product in the fully informative case. For deliberation cost satisfying $c \leq \frac{1}{\beta} \frac{\alpha}{1-\alpha}\left(\theta_{h}-\theta_{l}\right)\left[\alpha\left(\beta^{h}-\beta\right) \theta_{h}+(1-\alpha)\left(\beta^{l}-\beta\right) \theta_{l}\right]$, if $\alpha \in\left(\max \left\{1-\frac{1}{\frac{\beta^{h}\left(\beta-\beta^{l}\right)}{\beta(1-\beta)}}, \frac{\frac{\beta^{h}\left(\beta-\beta^{l}\right)}{\beta(1-\beta)}}{1+\frac{\beta^{h}\left(\beta-\beta^{l}\right)}{\beta(1-\beta)}}\right\}, 1\right), q_{l} \geq q_{l}^{f}$. For deliberation cost satisfying $c>\frac{1}{\beta} \frac{\alpha}{1-\alpha}\left(\theta_{h}-\theta_{l}\right)\left[\alpha\left(\beta^{h}-\beta\right) \theta_{h}+\right.$ $\left.(1-\alpha)\left(\beta^{l}-\beta\right) \theta_{l}\right]$, given any $\alpha \in(0,1), q_{l}<q_{l}^{f}$. In the fully informative case, $q_{l}^{f}$ takes the interior solution for $c \leq \bar{c}^{f}$ and the boundary solution for $c>\bar{c}^{f}$ (Guo and Zhang, 2012).

In the product line design with quantal preferences, the quality of low-type product is always below the efficient level. This fact shows that for dealing with the low-type product, a firm's dominant concern is always preventing high preference consumers from mimicing the low preference consumers. However, due to the quantal preferences, the strength of the sorting effect exerted by $q_{l}$ varies with respect to deliberation cost. It can be weaker $\left(q_{l}>q_{l}^{f}\right)$ or stronger $\left(q_{l}<q_{l}^{f}\right)$ than in the fully informative case.

In particular, Proposition 5 shows that whether the quality of the low-type product can be greater than its counterpart in the fully informative case depends on the prior belief. A sufficient condition to ensure $q_{l} \geq q_{l}^{f}$ is provided in Proposition 5. When high preference consumers generically take a high enough proportion such that $\alpha \in\left(\max \left\{1-\frac{1}{\frac{\beta^{h}\left(\beta-\beta^{l}\right)}{\beta(1-\beta)}}, \frac{\frac{\beta^{h}\left(\beta-\beta^{l}\right)}{\beta(1-\beta)}}{1+\frac{\beta^{h}\left(\beta-\beta^{l}\right)}{\beta(1-\beta)}}\right\}, 1\right)$, $q_{l} \geq q_{l}^{f}$ happens for deliberation cost below or equal to $\frac{1}{\beta} \frac{\alpha}{1-\alpha}\left(\theta_{h}-\theta_{l}\right)\left[\alpha\left(\beta^{h}-\beta\right) \theta_{h}+(1-\alpha)\left(\beta^{l}-\beta\right) \theta_{l}\right]$. It indicates that the sorting effect in this situation is weaker than in the fully informative case.

While for $c>\frac{1}{\beta} \frac{\alpha}{1-\alpha}\left(\theta_{h}-\theta_{l}\right)\left[\alpha\left(\beta^{h}-\beta\right) \theta_{h}+(1-\right.$ $\left.\alpha)\left(\beta^{l}-\beta\right) \theta_{l}\right]$, deliberation is costly and all consumers prefer doing it less. The posterior beliefs become less precise and hence perceived preferences are hard to be reliable to use for making decisions. In such a situation, a rational decision maker can only expect their preference information and form their expectations by using the posterior beliefs, which contain information about underlying preferences. Therefore, consumers would be more prone to make purchasing decisions by referring to the expected preference $\frac{\alpha \beta^{h} \theta_{h}+(1-\alpha) \beta^{l} \theta_{l}}{\beta}$ and $\frac{\alpha\left(1-\beta^{h}\right) \theta_{h}+(1-\alpha)\left(1-\beta^{l}\right) \theta_{l}}{1-\beta}$ in this situation. Therefore, the concern of limiting the surplus losses for the high preference consumers who perceive a low preference after deliberation becomes weaker, which in turn makes incentive compatibility concern more crucial than when the deliberation cost is low. Therefore, sorting effect becomes stronger. Thus, in this situation, for any $\alpha \in(0,1), q_{l}<q_{l}^{f}$.

Proposition 6: In the product line design with quantal preferences, for $c<\bar{c}$, the price of the high-type product is strictly greater than the price of the low-type product if and only if the distance between the valuation of high preference and low preference is large enough, i.e., $p_{h}>p_{l}$ if and only if $\theta_{h}>$ $\frac{-\left[(1-\alpha) \beta^{l}-\alpha \beta^{h}\right] \theta_{l}+\sqrt{\left\{\left[(1-\alpha) \beta^{l}-\alpha \beta^{h}\right]^{2}+4 \alpha(1-\alpha) \beta^{h} \beta^{l}\right\} \theta_{l}^{2}+\frac{4 \alpha \beta^{h}}{\phi}} c}{2 \alpha \beta^{h}}$. For $c \geq \bar{c}$, the price of the high-type product is always strictly greater than the price of the low-type product.

$\frac{\underset{-\left[(1-\alpha) \beta^{l}-\alpha \beta^{h}\right] \theta_{l}+\sqrt{\left\{\left[(1-\alpha) \beta^{l}-\alpha \beta^{h}\right]^{2}+4 \alpha(1-\alpha) \beta^{h} \beta^{l}\right\} \theta_{l}^{2}+\frac{4 \alpha \beta^{h}}{\phi}} c}{2 \alpha \beta^{h}}}{\operatorname{cor}}$, low type product is not produced.

In the fully informative case, $p_{h}>p_{l}$ is held automatically. But after introducing quantal preferences, $p_{h}>p_{l}$ can only be held when certain conditions are satisfied. Proposition 6 implies that if the difference between high preference and low preference is not large enough for $c<\bar{c}$, although $\theta_{h}>\theta_{l}$, $p_{h}<p_{l}$ happens. Although the direct result from this product line design is providing both high-type and low-type products to consumers, all consumers will buy the high quality product even without deliberation in this situation. In practice, it means there is a waste by producing the low-type product. Hence, in this situation, the product line has to be redesigned by only serving the high-type product. Therefore, we obtain Corollary 3.

$p_{h}>p_{l}$ is a reflection of the incentive compatibility requirement such that consumers conform to their perceived preferences and do not mimic the other type of consumers. The possibility that $p_{h}<p_{l}$ when $c<\bar{c}$ indicates that in some situations, the incentive compatibility requirements are hard to achieve. Proposition 6 shows that such unusual situations happen when $\theta_{h}$ and $\theta_{l}$ are close enough. As a result of deliberation, a reshuffling of consumers happens between the populations of the two types of consumers according to their true preferences. The closer the preferences $\theta_{h}$ and $\theta_{l}$ are, then after deliberation, the closer the average preferences of each new-formed consumer groups according to the perceived preferences, and hence the incentive compatibility constraints $I C_{h}^{d}$ and $I C_{l}^{d}$ that motivate consumers to truth-tell their perceived preferences are more difficult to perform the function 
of sorting. It can even backfire such that the product line that aims sorting out different types of consumers fails to do so. That is, $p_{h}<p_{l}$. Therefore, to design a product line that incentivizes consumers to truth-tell according to their perceived preferences, the difference between the valuation of high preference and low preference has to be large enough. Otherwise, the firm just needs to design a product line to provide high-type product. Therefore, quantal preferences bring a more demanding requirement on consumer preferences to design a screening contract.

For $c \geq \bar{c}$, both consumers perceiving a high preference and consumers perceiving a low preference are indifferent between the high-type product and the low-type product in equilibrium. Therefore, the incentive compatibility consideration is not as important as in the case of $c<\bar{c}$. In this situation, the result $p_{h}>p_{l}$ is obtained mainly due to the firm's profit maximization purpose: for $c \geq \bar{c}$, deliberation becomes costly and hence average preference becomes more crucial for the firm as a reference to design the product line. For maximizing profit, the designed product line in equilibrium makes consumers with high (low) average preference buy the high (low)-type product. The average preference of consumers perceiving a high preference is greater than its counterpart of consumers perceiving a low preference, i.e. $\frac{\alpha \beta^{h} \theta_{h}+(1-\alpha) \beta^{l} \theta_{l}}{\beta}>\frac{\alpha\left(1-\beta^{h}\right) \theta_{h}+(1-\alpha)\left(1-\beta^{l}\right) \theta_{l}}{1-\beta}$. Therefore, at equilibrium, the high type product is priced more expensive than the low-type product so that the prices are consistent with the average preferences of each group of consumers.

Proposition 6 and hence Corollary 3 show that the low-type product cannot be produced in some situations due to a distortion of the incentives of sorting out the two types of consumers. Another reason that low-type product is not produced can be that its quality may be negative. In the fully informative case, the quality of low type product can also be negative (Guo and Zhang, 2012). However, when we consider the quantal consumer preferences, it brings additional requirement on preferences for obtaining the same result compared with that in the fully informative case. Lemma 3 formally states this result.

Lemma 3: For the deliberation cost below $\bar{c}$, the quality of low-type product is positive if and only if $\frac{\theta_{l}}{\theta_{h}}>-\frac{\frac{\alpha \beta^{h}\left(\beta^{l}-\beta\right)}{\beta(1-\beta)}}{1-\frac{\alpha \beta^{h}\left(\beta^{l}-\beta\right)}{\beta(1-\beta)}}$, i.e. $\theta_{h}$ and $\theta_{l}$ are close enough. For the deliberation cost greater than or equal to $\bar{c}$, the quality of low-type product is positive if and only if $\theta_{h}>\frac{2 \sqrt{(1-\alpha)\left(\beta-\beta^{l}\right) \beta c}}{\alpha\left(\beta^{h}-\beta\right)}$ and $\frac{2\left(\beta^{h}-\beta\right) \theta_{h}-\sqrt{\alpha^{2}\left(\beta^{h}-\beta\right)^{2} \theta_{h}^{2}-4(1-\alpha)\left(\beta-\beta^{l}\right) \beta c}}{2(1-\alpha)\left(\beta-\beta^{l}\right)}<\theta_{l}<$ $\frac{2\left(\beta^{h}-\beta\right) \theta_{h}+\sqrt{\alpha^{2}\left(\beta^{h}-\beta\right)^{2} \theta_{h}^{2}-4(1-\alpha)\left(\beta-\beta^{l}\right) \beta c}}{2(1-\alpha)\left(\beta-\beta^{l}\right)}$, i.e., the high preference is large enough and the difference between high preference and low preference is also large enough. $\square$

In the fully informative case, when $c<\bar{c}^{f}$, where $\bar{c}^{f}=\alpha\left(\theta_{h}-\theta_{l}\right)^{2}, q_{l}>0$, but when $c \geq \bar{c}^{f}, q_{l}>0$ if $c<(1-\alpha) \theta_{l}\left(\theta_{h}-\theta_{l}\right)$, supposing $\bar{c}^{f}<(1-\alpha) \theta_{l}\left(\theta_{h}-\theta_{l}\right)$
(Guo and Zhang, 2012). Whether $q_{l}>0$ in the fully informative case is only up to the deliberation cost. In our model, the quantal preference changes the essence that ensures $q_{l}>0$ compared with that in the fully informative case. Except a requirement on deliberation cost, it puts additional requirement on consumer preferences to determine whether $q_{l}$ is greater than zero or not. Note that if the quality of a certain type of product is negative, then the price of this type of product is also negative given that the quality of the other type of product is non-negative, so is the profit of this type of product.

Proposition 7: Suppose the conditions and results in Lemma 3 are satisfied. For a deliberation cost strictly smaller than $\bar{c}$, the consumers whose true preference is low and buy the low-type product get a positive surplus, i.e., $\theta_{l} q_{l}>p_{l}$, if and only if $\theta_{h}-\theta_{l}>\sqrt{\frac{c}{\alpha \beta^{h} \phi}}$, i.e., the difference between the valuation of high preference and low preference is large enough. For a deliberation cost greater than or equal to $\bar{c}$, $\theta_{l} q_{l}>p_{l}$ is always held.

In any screening contract, including the fully informative case of Guo and Zhang (2012), low-preference consumers get no surplus in equilibrium, i.e. $\theta_{l} q_{l}-p_{l}=0$. As we discussed in the last section, the product line design mechanism that considers the quantal preferences will make the firm sacrifice part of its profit to compensate consumers whose perceived preference is incorrect after deliberation. In addition, consumers who correctly perceived their types will also get more surplus than the fully informative case to ensure that the population of a specific type of consumers as a whole are willing to participate the purchasing activity. The non-negative surplus at equilibrium $\theta_{l} q_{l}-p_{l}>0$ justifies this intuition. For the deliberation cost below $\bar{c}, \theta_{l} q_{l}<p_{l}$ for $\theta_{h}-\theta_{l}<\sqrt{\frac{c}{\alpha \beta^{h} \phi}}$. In this situation, the expected surplus $\beta^{l}\left(\theta_{l} q_{h}-p_{h}\right)+\left(1-\beta^{l}\right)\left(\theta_{l} q_{l}-p_{l}\right)<0$, and therefore the product line design problem should be reformulated so that only high-type product is produced to serve consumers.

Define $\pi_{h}=p_{h}-\frac{q_{h}^{2}}{2}$, which is the firm's profit for selling the high-type product, and $\pi_{l}=p_{l}-\frac{q_{l}^{2}}{2}$, which is the firm's profit for selling the low-type product. If the high-type product is not produced, a situation that does not happen in the fully informative case, it can be due to that $\pi_{h}$ is negative. Unlike the fully informative case, or any conventional binary type screening contract, a positive $\pi_{h}$ requires certain conditions to support it:

Proposition 8: Given any deliberation cost $0<c<$ $\frac{2\left[\frac{\alpha \beta^{h}\left(1-\beta^{l}\right)}{\beta} \theta_{h}+\beta^{l}\left[1+\frac{(1-\alpha)\left(1-\beta^{l}\right)}{\beta}\right] \theta_{l}\right]^{2}}{1-\beta^{l}}$, a positive $\pi_{h}$ can be obtained if and only if $A_{h}-\sqrt{A_{h}^{2}+\left(2 B_{h} q_{l}-\frac{1-\beta^{l}}{2} c\right)}<$ $q_{h}<A_{h}+\sqrt{A_{h}^{2}+\left(2 B_{h} q_{l}-\frac{1-\beta^{l}}{2} c\right)}$ and $q_{l}<$ $\frac{\left[\frac{\alpha \beta^{h}\left(1-\beta^{l}\right)}{\beta} \theta_{h}+\beta^{l}\left[1+\frac{(1-\alpha)\left(1-\beta^{l}\right)}{\beta}\right] \theta_{l}\right]^{2}-\frac{\left(1-\beta^{l}\right) c}{2}}{2\left(\theta_{h}-\theta_{l}\right) \frac{\alpha \beta^{h}\left(1-\beta^{l}\right)}{\beta}}$. 
Corollary 4: If the deliberation cost is strictly greater than $\frac{2\left[\frac{\alpha \beta^{h}\left(1-\beta^{l}\right)}{\beta} \theta_{h}+\beta^{l}\left[1+\frac{(1-\alpha)\left(1-\beta^{l}\right)}{\beta}\right] \theta_{l}\right]^{2}}{1-\beta^{l}}$, then $\pi_{h}$ is non-positive.

$\pi_{h}$ is a quadratic function with respect to $q_{h}$. Therefore, the non-negative profit can only be taken when $q_{h}$ is valued at certain range. Proposition 8 shows that the range of $q_{h}$ that produces the positive $\pi_{h}$ depends on $q_{l}$. The larger $q_{l}$ it is, the narrower the range is. To guarantee the non-negative profit for selling the high-type product, the value of $q_{l}$ cannot be larger than a certain level. Intuitively, consumers whose perceived preference is high have stronger willingness to consume both types of products, and therefore the low-type product can share the demand from consumers who perceive a high preference. The presence of quantal preferences reinforces the phenomenon that high preference consumers buy the low-type product since there are some high preference consumers mistakenly perceiving themselves having a low preference and honestly buy the low-type products according to their perceived preferences. Therefore, the quantal preferences make the low-type product more attractive, in terms of the more diversified consumer groups according to their perceived preferences, than in the fully informative case.

Proposition 8 also shows that the larger the deliberation cost it is, the smaller value range of $q_{l}$ and $q_{h}$ leading to a positive $\pi_{h}$. For consumers whose perceived preference is high, which is above their expected preference $\theta_{m}$, they are more willing to buy the high-type product by deliberation than without making deliberation. The surplus these consumers expect to get according to their perceived preference by buying the high-type product, i.e., $\theta_{h} q_{h}-p_{h}$, is greater than the obtained surplus according to the expected preference without making deliberation, i.e., $\theta_{m} q_{h}-p_{h}$, and this expectation is also reflected by the establishment of the $I C^{d}$ constraint. The smaller the deliberation cost it is, the more willing consumers are to deliberate, and hence the more likely a positive $\pi_{h}$ is obtained, which result further confirms for these consumers and the firm that deliberation and motivating it was worthwhile for them. When deliberation is too costly, this range collapses so that no $q_{l}$ and $q_{h}$ obtained by motivating consumers to deliberate can lead to a positive $\pi_{h}$.

When quantal preferences are considered, the necessary and sufficient condition that ensures $\pi_{l}$ positive is as follows.

Proposition 9: For any $c>0$, a positive $\pi_{l}$ can be obtained if and only if $B_{l}-\sqrt{B_{l}^{2}+2\left[A_{l} q_{h}+\frac{\beta^{l}}{\beta} c\right]}<q_{l}<$ $B_{l}+\sqrt{B_{l}^{2}+2\left[A_{l} q_{h}+\frac{\beta^{l}}{\beta} c\right]}$ and $q_{h}<-\frac{B_{l}^{2}+2 \frac{\beta^{l}}{\beta} c}{2 A_{l}}$.

$\pi_{l}$ is a quadratic function with respect to $q_{l}$. Therefore, the non-negative profit can only be taken when $q_{l}$ is valued at restricted range. The range of $q_{l}$ that produces a positive $\pi_{l}$ depends on $q_{h}$. The larger $q_{h}$ it is, the narrower the range is. To guarantee the non-negative profit, the value of $q_{h}$ is limited and cannot be high. This is because compared with the fully informative case, the presence of quantal preferences makes the high-type product influence the low-type product's pricing, a fact that can be found from equation 2 . A high $q_{h}$ will reduce the price of low type product and hence a negative $\pi_{l}$ is more likely to happen.

Contrary to the case for the high-type product, according to Proposition 9, the larger the deliberation cost it is, the broader range of $q_{l}$ and $q_{h}$ leading to a positive $\pi_{l}$ it is. Hence, a positive $\pi_{l}$ is more facilitated to achieve in this situation. Intuitively, consumers whose perceived preference is low, which is below the expected preference $\theta_{m}$, are less willing to buy the low-type product by deliberation than without making deliberation. This intuition can be confirmed by the relationship that the surplus for consumers who perceive themselves as low preference expected to obtain, i.e., $\theta_{l} q_{l}-p_{l}$, is smaller than the surplus for consumers who purchase the product according to the expected preference, i.e., $\theta_{m} q_{l}-p_{l}$. Proposition 9 implies that there exists a possibility that consumers whose perceived preference is low regret deliberation after finding out their true preference is low. Therefore, from a different perspective, Proposition 9 also shows that consumers whose true preference is low are more prone to make purchasing decisions by referring to the expected preference $\theta_{m}$ while making no deliberation. Therefore, the larger the deliberation cost, the more likely consumers make purchasing decisions by referring to the expected preference $\theta_{m}$ by making no deliberation, and hence the posterior beliefs become less sensitive to deliberation cost once deliberation is made and consumers rely on the expected preferences $\frac{\alpha \beta^{h} \theta_{h}+(1-\alpha) \beta^{l} \theta_{l}}{\beta}$ and $\frac{\alpha\left(1-\beta^{h}\right) \theta_{h}+(1-\alpha)\left(1-\beta^{l}\right) \theta_{l}}{1-\beta}$ to make decisions. It is known that for small deliberation cost, consumers are more likely to find out their true preferences and hence a positive $\pi_{l}$ is more likely to obtain. However, as posterior beliefs are less sensitive to the deliberation cost as it increases, $\pi_{l}$ tends to keep positive during this process according to equation 1 to 4 . Therefore, the product line can still bring a positive $\pi_{l}$ even if deliberation cost is large.

\section{Conclusion}

Quantal preference is a description of the phenomenon that consumers may not perceive their true preferences after a cognitive process. The cognitive process considered in our paper is thinking, according to the subject classification in cognitive psychology, or equivalently deliberation, as it is called by Guo and Zhang (2012). The quantal preferences are consistent with the experimental findings in economics literature that consumers' stated preferences may be erroneous or imprecise and hence cannot accurately reflect consumers' true preferences. Expectedly, the quantal preferences can be obtained through various theories. In our paper about designing a product line with quantal consumer preferences, we focus on deriving the common features and properties of the product line brought by the quantal consumer preferences, no matter how the quantal consumer preferences are derived and as a result of any theory. 
In this paper's analysis of designing a product line by considering quantal consumer preferences, one major insight we can draw is that a marketing strategy that helps consumers find out their true preferences essentially helps the firm itself to gain a larger profit. First, the firm's profit of each type of product reaches its largest when the deliberation cost equals zero. This is because when the deliberation cost equals zero, consumers can accurately find out their true preferences and make the solution set that is framed by the constraints of the product design problem largest. Second, the firm has to subsidize consumers for limiting their potential surplus losses due to a wrong perception of their own preference by deliberation, and to subsidize each type of consumers, the firm has to sacrifice part of its profit. If there is no mistake for consumers upon finding out their true preferences by deliberation, as in the fully informative case of Guo and Zhang (2012), consumers will not suffer a surplus loss, and the firm would not need to sacrifice part of its profits to limit those consumers' surplus losses. Hence, the more clearly that consumers can recognize their true preferences, the more both the firm's profit and consumers' welfare can be improved. Therefore, a societal welfare improvement can be realized in a behavioral way. Third, as revealed by our analysis, the product line design that considers the quantal consumer preferences is very complicated and flexible. The equilibrium product line exhibited in our model is derived under Assumption 2, which is a requirement on the posterior beliefs. The way of deriving an equilibrium, the equilibrium itself, and its properties, can also be changed under different requirements on the posterior beliefs. However, if the consumers can clearly find out their true preferences as much as possible, and hence make the product line design problem closer to the fully informative setting like the one considered by Guo and Zhang (2012), the product line design problem can be much simplified.

This paper has a general impact on the theory of screening contract. In contract theory, the approach to obtaining a screening contract is standard and the contracts' equilibrium properties are also regular. Most screening contracts studied in existing literature is of binary type, and some standard conclusions for binary-type screening contract can be extended to multiple-type screening contract (Bolton and Dewatripont, 2005). In this paper, quantal preferences are introduced into a standard binary-type screening contract (the product line), and we find that most standard results in conventional screening contracts are changed. First of all, the approaches to obtaining a screening contract become flexible. Which approach will be adopted depends on the particular parameter specifications when formulating the product line (contract) design problem. We adopt a parameter specification that follows Assumption 2, and the standard approach for obtaining a screening contract for conventional binary type contract can be again used to obtain a screening contract when quantal preferences are considered (Proposition 1).

For a screening contract obtained subject to Assumption 2 , the following properties for conventional binary-type screening contracts are kept even if quantal preferences are introduced: only separating equilibrium exists, high preference consumers get positive surplus by choosing high type product, and the quality of low type product is below the efficient level (Proposition 2). We also obtain some interesting results that are in contrast to those in the classical product line design, and they provide some new managerial insights. The quality of high type product could be greater or smaller than the efficient level, while in standard binary-type screening contract it is always kept at the efficient level (Proposition 4; Bolton and Dewatripont, 2005). The price of high type product is always greater than the price of low type product under any circumstance in standard binary-type screening contract. However, if quantal preferences are considered, this conclusion can only be held when certain conditions are satisfied (Bolton and Dewatripont, 2005; Proposition 6). In conventional binary-type screening contract, it is possible that the production of low type product is ceased, but it is impossible for the high type product (Bolton and Dewatripont, 2005). However, when we consider the quantal preferences, this standard result is no longer held. Proposition 8 and Corollary 4 show that the profit of high type product can be negative and hence it cannot be produced. Besides, the quality of the high type product can be negative and hence the high type product is not produced (Corollary 2). Of course, it is still possible that the low type product cannot be produced under some situations (Corollary 3, Lemma 3, Proposition 7, Proposition 9).

Cases of the product line design problem if Assumption 2 is not held have different solving approaches. Except the case where $\beta^{h}>\frac{1}{2}$ and $\beta^{l}<\frac{1}{2}$, but $1-\beta^{l}<\beta^{h}$, all remaining cases have different formulations of the reduced problems from the case where Assumption 2 is held (recall how we obtain the reduced form of the product line design problem where Assumption 2 is held). For the case where $\beta^{h}>\frac{1}{2}$ and $\beta^{l}<\frac{1}{2}$, but $1-\beta^{l}<\beta^{h}$, the formulation of the reduced problem is exactly the same as the one subject to Assumption 2. However, in this case, we cannot obtain the relationship such that $P C^{l}$ and $I C^{h}$ imply $P C^{h}$. Therefore, the equilibrium product line in this case will have different properties from its counterpart where Assumption 2 is held in this paper. For the case where $\beta^{h}>\frac{1}{2}$ and $\beta^{l}>\frac{1}{2}$ and the case where $\beta^{h}<\frac{1}{2}$ and $\beta^{l}>\frac{1}{2}$, because $\theta^{h}>\theta^{l}$, extra conditions are required to make the relationship $P C^{l}$ and $I C^{h}$ implies $P C^{h}$ happen. Therefore, due to the extra conditions, even if the relationship that $P C^{l}$ and $I C^{h}$ implies $P C^{h}$ is held, the equilibrium product line in these two cases will have different properties from the case where Assumption 2 is held. Finally, for the case where $\beta^{h}>\frac{1}{2}$ and $\beta^{l}<\frac{1}{2}$, the relationship that $P C^{l}$ and $I C^{h}$ implies $P C^{h}$ does not happen under any circumstance. All above results about the remaining cases are obtained based on the proof of Proposition 1. The relationship that $P C^{l}$ and $I C^{h}$ implies $P C^{h}$ is crucial for reducing and solving the product line design problem in our case, and it is also the core of the conventional approach for solving any screening contract, for example the fully informative case by Guo and Zhang (2012). Therefore, except the case where As- 
sumption 2 is held, in any other case, this relationship is either not held or requiring additional conditions or constraints to make them held, both of which determine that the equilibrium product line in these cases will have different properties from the case where Assumption 2 is held, and are less suitable to compare with the fully informative case than the case subject to Assumption 2. However, even though they are less suitable to compare with the fully informative case, the remaining cases are also interesting and worthwhile for further study. Studying the equilibrium properties of the product line where Assumption 2 is not held will be a meaningful extension of this paper.

This paper also provides a behavioral explanation on contract incompleteness. The contract incompleteness where the state information (preference information in our context) is hard to verify can arise due to human beings' own limitation in their psychological or cognitive activities. In the product line design with quantal preferences, due to its property that the firm and consumers only interact once, i.e., the firm and consumers only interact in the one-shot purchasing activity, its analysis is based on the complete contract framework. In a contract design problem where the firm and consumers can interact more than once, for example the types of consumer preferences are more than two and an additional purchasing activity where deliberation becomes more costly is allowed to happen between the firm and consumers who perceive a wrong preference by deliberation in the last round of purchasing activity, such a problem, where preference information is unverifiable, can be analyzed in the incomplete contract framework. Technically, our paper is an example to show that a contract design with unverifiable states can still be dealt within the framework of complete contract theory.

As revealed by this study, improving consumers' deliberation ability to correctly find out their true preferences, i.e. reducing the deliberation cost, can be beneficial for both the firm and consumers, if the firm and consumers interact only once. If the transaction process can be more structural than just a one-shot purchasing activity between the firm and consumers, e.g., adding some ex post stages such that consumers who correctly find out their true preferences share the additional surplus relative to the efficient level with consumers of the same type but who perceive a wrong preference and hence suffer a surplus loss, intuitively it is possible that the firm can subsidize less to motivate the population of each type of consumers to participate the purchasing activity. If we upgrade the transaction process considered in our paper to a complex one structured like this, the problem of product line design with quantal consumer preferences can be analyzed by the methodology of incomplete contract theory. Besides, a further and more extensive societal welfare improvement is also intuitively promising if we extend our model in this way.

\section{Appendix}

\section{A Proof of Proposition 1}

Proof: Given that $\theta_{h}>\theta_{l}, I C^{h}$ implies the following relationship: $\beta^{h}\left(\theta_{h} q_{h}-p_{h}\right)+\left(1-\beta^{h}\right)\left(\theta_{h} q_{l}-\right.$ $\left.p_{l}\right) \geq\left(1-\beta^{h}\right)\left(\theta_{h} q_{h}-p_{h}\right)+\beta^{h}\left(\theta_{h} q_{l}-p_{l}\right)>$ $\left(1-\bar{\beta}^{h}\right)\left(\theta_{l} q_{h}-p_{h}\right)+\beta^{h}\left(\theta_{l} q_{l}-p_{l}\right)$. If $\left(1-\beta^{h}\right)\left(\theta_{l} q_{h}-\right.$ $\left.p_{h}\right)+\beta^{h}\left(\theta_{l} q_{l}-p_{l}\right) \geq \beta^{l}\left(\theta_{l} q_{h}-p_{h}\right)+\left(1-\beta^{l}\right)\left(\theta_{l} q_{l}-p_{l}\right)$, i.e. $\left(1-\beta^{h}-\beta^{l}\right)\left(\theta_{h} q_{h}-p_{h}\right) \geq\left(1-\beta^{h}-\beta^{l}\right)\left(\theta_{l} q_{l}-p_{l}\right)$, then constraints $P C^{l}$ and $I C^{h}$ imply $P C^{h}$ and this condition can be equivalently transformed to:

$$
\left\{\begin{array}{l}
\beta^{h}+\beta^{l} \leq 1 \\
\theta_{h} q_{h}-p_{h} \geq \theta_{l} q_{l}-p_{l}
\end{array}\right.
$$

and

$$
\left\{\begin{array}{l}
\beta^{h}+\beta^{l} \geq 1 \\
\theta_{h} q_{h}-p_{h} \leq \theta_{l} q_{l}-p_{l}
\end{array}\right.
$$

For the latter case, because given that $\beta^{h}>\frac{1}{2}, I C^{h}$ is equivalent to $\theta_{h} q_{h}-p_{h} \geq \theta_{h} q_{l}-p_{l}$ and hence $\theta_{h} q_{h}-p_{h}>\theta_{l} q_{l}-p_{l}$, therefore it contradicts against $I C^{h}$ and not valid in this setting. Because $\theta_{h}>\theta_{l}$, hence $\theta_{h} q_{h}-p_{h}>\theta_{l} q_{l}-p_{l}$. Therefore, $P C^{l}$ and $I C^{h}$ imply $P C^{h}$ if and only if $1-\beta^{l} \geq \beta^{h}$. Therefore, Proposition 1 is proven.

\section{B Equilibrium Product Line that Both Types of Consumers are Served}

Given Assumption 2, similar to the benchmark case, because $\theta_{m} q_{l}-p_{l} \geq \theta_{l} q_{l}-p_{l} \geq 0$, the RHS of $I C^{d}$ can be reduced to $\max \left(\theta_{m} q_{h}-p_{h}, \theta_{m} q_{l}-p_{l}\right)$. Specifically, the high-type product serves deliberated consumers better if

$$
\begin{gathered}
-c+\alpha \beta^{h}\left(\theta_{h} q_{h}-p_{h}\right)+\alpha\left(1-\beta^{h}\right)\left(\theta_{h} q_{l}-p_{l}\right)+(1-\alpha) \beta^{l}\left(\theta_{l} q_{h}-p_{h}\right) \\
+(1-\alpha)\left(1-\beta^{l}\right)\left(\theta_{l} q_{l}-p_{l}\right) \geq \theta_{m} q_{h}-p_{h}
\end{gathered}
$$

or equivalently

$$
\begin{gathered}
p_{h}-p_{l} \geq \frac{\alpha\left(1-\beta^{h}\right) \theta_{h}+(1-\alpha)\left(1-\beta^{l}\right) \theta_{l}}{1-\beta}\left(q_{h}-q_{l}\right) \\
+\frac{c}{1-\beta}\left(I C_{h}^{d}\right)
\end{gathered}
$$

The low-type product serves deliberated consumers better if

$$
-c+\alpha \beta^{h}\left(\theta_{h} q_{h}-p_{h}\right)+\alpha\left(1-\beta^{h}\right)\left(\theta_{h} q_{l}-p_{l}\right)+(1-\alpha) \beta^{l}\left(\theta_{l} q_{h}-p_{h}\right)
$$




$$
+(1-\alpha)\left(1-\beta^{l}\right)\left(\theta_{l} q_{l}-p_{l}\right) \geq \theta_{m} q_{l}-p_{l}
$$

or equivalently

$$
p_{h}-p_{l} \leq \frac{\alpha \beta^{h} \theta_{h}+(1-\alpha) \beta^{l} \theta_{l}}{\beta}\left(q_{h}-q_{l}\right)-\frac{c}{\beta} \quad\left(I C_{l}^{d}\right)
$$

Given $I C^{h^{\prime}}, I C_{h}^{d}$ implies $I C^{l^{\prime}}$, and given $I C^{l^{\prime}}, I C_{l}^{d}$ implies $I C^{h^{\prime}}$. Therefore, both $I C^{h^{\prime}}$ and $I C^{l^{\prime}}$ are simultaneously replaced by $I C_{h}^{d}$ and $I C_{l}^{d}$. At equilibrium, $I C_{l}^{d}$ is binding. Otherwise, $p_{h}$ and $p_{l}$ can be increased to improve the firm's profit while the constraints are still maintained. Therefore, the optimal prices can be obtained from the two binding constraints $P C^{l}$ and $I C_{l}^{d}$. They are

$$
\begin{gathered}
p_{h}=A_{h} q_{h}+B_{h} q_{l}-\frac{\left(1-\beta^{l}\right) c}{\beta} \\
p_{l}=A_{l} q_{h}+B_{l} q_{l}+\frac{\beta^{l} c}{\beta}
\end{gathered}
$$

where $A_{h}=\frac{\alpha \beta^{h}\left(1-\beta^{l}\right)}{\beta} \theta_{h}+\beta^{l}\left[1+\frac{(1-\alpha)\left(1-\beta^{l}\right)}{\beta}\right] \theta_{l}$, $B_{h}=-\left(\theta_{h}-\theta_{l}\right) \frac{\alpha \beta^{h}\left(1-\beta^{l}\right)}{\beta}, A_{l}=-\left(\theta_{h}-\theta_{l}\right) \frac{\alpha \beta^{h} \beta^{l}}{\beta}$, and $B_{l}=\frac{\alpha \beta^{h} \beta^{l}}{\beta} \theta_{h}+\left(1-\frac{\alpha \beta^{h} \beta^{l}}{\beta}\right) \theta_{l}$. Given the obtained $p_{h}$ and $p_{l}$, the product line design problem by motivating consumers to deliberate can be transformed to:

$$
\max _{q_{h}, q_{l}} \beta\left(p_{h}-\frac{q_{h}^{2}}{2}\right)+(1-\beta)\left(p_{l}-\frac{q_{l}^{2}}{2}\right)
$$

s.t.

$$
q_{h}-q_{l} \geq \frac{c}{\alpha\left(\beta^{h}-\beta\right) \theta_{h}+(1-\alpha)\left(\beta^{l}-\beta\right) \theta_{l}}
$$

There exists a threshold of the deliberation cost $\bar{c}$ where $\bar{c}=\frac{\alpha \beta^{h}\left(\beta-\beta^{l}\right)}{\beta^{2}(1-\beta)}\left(\theta_{h}-\theta_{l}\right)\left[\alpha\left(\beta^{h}-\beta\right) \theta_{h}+(1-\alpha)\left(\beta^{l}-\beta\right) \theta_{l}\right]$. For $c \leq \bar{c}$, the equilibrium product qualities are the interior solutions of the above problem

$$
\begin{aligned}
& q_{h}=\frac{\alpha \beta^{h}}{\beta}\left(1-\frac{\beta^{l}}{\beta}\right) \theta_{h}+\left(\frac{\alpha \beta^{h}}{\beta^{2}}+\frac{1-\alpha}{\beta}\right) \beta^{l} \theta_{l} \\
& q_{l}=\frac{\alpha \beta^{h}\left(\beta^{l}-\beta\right)}{\beta(1-\beta)} \theta_{h}+\left[1-\frac{\alpha \beta^{h}\left(\beta^{l}-\beta\right)}{\beta(1-\beta)}\right] \theta_{l}
\end{aligned}
$$

For $c>\bar{c}$, the constraint in the above problem is binding and the equilibrium product qualities are the constrained solutions of the problem. They are $q_{h}=\theta_{l}+\frac{(1-\beta) c}{\alpha\left(\beta^{h}-\beta\right) \theta_{h}+(1-\alpha)\left(\beta^{l}-\beta\right) \theta_{l}}$, and $q_{l}=\theta_{l}-\frac{\beta c}{\alpha\left(\beta^{h}-\beta\right) \theta_{h}+(1-\alpha)\left(\beta^{l}-\beta\right) \theta_{l}}$.

\section{Proof of Proposition 2}

Proof: According to equation 3 and $4, \Delta q=\phi\left(\theta_{h}-\theta_{l}\right)$, where $\phi=\frac{\alpha \beta^{h}}{\beta}\left(1-\frac{\beta^{l}}{\beta}-\frac{\beta^{l}-\beta}{1-\beta}\right)$. Because $\frac{\alpha \beta^{h}}{\beta}\left(1-\frac{\beta^{l}}{\beta}-\frac{\beta^{l}-\beta}{1-\beta}\right)>0$, therefore $\Delta q>0$ is always held. The equilibrium is always separating.

$q_{l}<\theta_{l}$ can be directly observed from the interior solution and boundary solution of $q_{l}$. In the following, we prove $\theta_{h} q_{h}-p_{h}>0$. Given Assumption 1, $\theta_{h} q_{h}-p_{h}>\theta_{l} q_{l}-p_{l}$. Because in the quantal preference model, consumers who correctly perceive their types will get additional surpluses relative to the fully informative case, therefore $\theta_{l} q_{l}-p_{l}>0$. Therefore, $\theta_{h} q_{h}-p_{h}>0$ at equilibrium in the product line with quantal preferences.

\section{Proof of Lemma 1}

Proof: Because $\frac{\alpha \beta^{h}}{\beta}\left(1-\frac{\beta^{l}}{\beta}\right)<1$, therefore $\bar{c}=\frac{\alpha \beta^{h}\left(\beta-\beta^{l}\right)}{\beta^{2}(1-\beta)}\left(\theta_{h}-\theta_{l}\right)\left[\alpha\left(\beta^{h}-\beta\right) \theta_{h}+(1-\right.$ $\left.\alpha)\left(\beta^{l}-\beta\right) \theta_{l}\right]<\frac{\left(\theta_{h}-\theta_{l}\right)\left[\alpha\left(\beta^{h}-\beta\right) \theta_{h}+(1-\alpha)\left(\beta^{l}-\beta\right) \theta_{l}\right]}{1-\beta}$. Because $\frac{\theta_{h}}{\theta_{l}}>1>\frac{\alpha\left(1-\frac{\beta^{l}-\beta}{1-\beta}\right)+\frac{\beta^{l}-\beta}{1-\beta}}{\alpha\left(1-\frac{\beta^{l}-\beta}{1-\beta}\right)}$, therefore $\bar{c}^{f}=\alpha\left(\theta_{h}-\theta_{l}\right)^{2}>\frac{\left(\theta_{h}-\theta_{l}\right)\left[\alpha\left(\beta^{h}-\beta\right) \theta_{h}+(1-\alpha)\left(\beta^{l}-\beta\right) \theta_{l}\right]}{1-\beta}$.

\section{E Proof of Proposition 3}

Proof: For $c \leq \bar{c}, q_{h}>0$ can be rearranged to $\frac{\alpha \beta^{h}}{\beta} \theta_{h}+\frac{(1-\alpha) \beta^{l}}{\beta} \theta_{l}>\frac{\alpha \beta^{h} \beta^{l}}{\beta^{2}}\left(\theta_{h}-\theta_{l}\right)$ and hence $\frac{\alpha \beta^{h} \theta_{h}+(1-\alpha) \beta^{l} \theta_{l}}{\theta_{h}-\theta_{l}}>\frac{\alpha \beta^{h} \beta^{l}}{\beta}$.

For $c>\bar{c}, q_{h}>0$ can be rearranged to $\frac{1}{\left(\alpha \beta^{h} \theta_{h}+(1-\alpha) \beta^{l} \theta_{l}\right)-\beta\left(\alpha \theta_{h}+(1-\alpha) \theta_{l}\right)}>-\frac{\theta_{l}}{(1-\beta) c}$. If $\frac{\alpha \beta^{h} \theta_{h}+(1-\alpha) \beta^{l} \theta_{l}}{\beta}>\alpha \theta_{h}+(1-\alpha) \theta_{l}, q_{h}>0$ is held. If $\frac{\alpha \beta^{h} \theta_{h}+(1-\alpha) \beta^{l} \theta_{l}}{\beta}<\alpha \theta_{h}+(1-\alpha) \theta_{l}, q_{h}>0$ can be further rearranged to $\frac{\alpha \beta^{h} \theta_{h}+(1-\alpha) \beta^{l} \theta_{l}}{\beta}<-\frac{(1-\beta) c}{\beta \theta_{l}}+\alpha \theta_{h}+(1-\alpha) \theta_{l}$.

Therefore, we obtain Proposition 3.

\section{F Proof of Proposition 4}

Proof: For deliberation cost $c<\bar{c}$, according to equation 3, $q_{h}$ is a weighted average of $\theta_{h}$ and $\theta_{l}$, and hence $q_{h}<\theta_{h}$.

For $\bar{c} \leq c<\frac{\left(\theta_{h}-\theta_{l}\right)\left[\alpha\left(\beta^{h}-\beta\right) \theta_{h}+(1-\alpha)\left(\beta^{l}-\beta\right) \theta_{l}\right]}{1-\beta}$, according to the boundary solution of $q_{h}, q_{h}<\theta_{h}$. For deliberation cost satisfying $\frac{\left(\theta_{h}-\theta_{l}\right)\left[\alpha\left(\beta^{h}-\beta\right) \theta_{h}+(1-\alpha)\left(\beta^{l}-\beta\right) \theta_{l}\right]}{1-\beta} \leq c<\bar{c}^{f}$, according to the boundary solution of $q_{h}, q_{h} \geq \theta_{h}$. It is because for $c>\bar{c}$, 
$q_{h} \leq \theta_{h}$ if and only if $c \leq \frac{\left(\theta_{h}-\theta_{l}\right)\left[\alpha\left(\beta^{h}-\beta\right) \theta_{h}+(1-\alpha)\left(\beta^{l}-\beta\right) \theta_{l}\right]}{1-\beta}$

For $c \geq \bar{c}^{f}$, the boundary solution $q^{h}>q^{h f}$. $q^{h f}$ equals $\theta_{l}+\frac{c}{\alpha\left(\theta_{h}-\theta_{l}\right)}$ and is the boundary solution in the fully informative case (Guo and Zhang, 2012). $q_{h}-q_{h}^{f}=\frac{\alpha\left(1-\beta^{h}\right)\left(\theta_{h}-\theta_{l}\right)}{\alpha\left(\theta_{h}-\theta_{l}\right)\left[\alpha\left(\beta^{h}-\beta\right) \theta_{h}+(1-\alpha)\left(\beta^{l}-\beta\right) \theta_{l}\right]} c$ and hence $q^{h}>q^{h f}$

\section{G Proof of Corollary 2}

Proof: recall equation 3. For $c<\bar{c}, q_{h}>0$ is equivalent to $\left[\frac{\alpha \beta^{h} \beta^{l}}{\beta^{2}}+\frac{(1-\alpha) \beta^{l}}{\beta}\right] \theta_{l}>-\left[\frac{\alpha \beta^{h}}{\beta}-\frac{\alpha \beta^{h} \beta^{l}}{\beta^{2}}\right] \theta_{h}$. By recollecting terms on both sides of this inequality, we obtain that $\frac{\theta_{l}}{\theta_{h}}>-\frac{\alpha \beta^{h}\left(1-\frac{\beta^{l}}{\beta}\right)}{\beta^{l}\left[\frac{\alpha \beta^{h}}{\beta}+(1-\alpha)\right]}$. For $c \geq \bar{c}$, because $\alpha\left(\beta^{h}-\beta\right) \theta_{h}+(1-\alpha)\left(\beta^{l}-\beta\right) \theta_{l}=\alpha(1-\alpha)\left(\beta^{h}-\beta^{l}\right)\left(\theta_{h}-\theta_{l}\right)>$ 0 under Assumption 2, therefore $q_{h}>0$ is always held. Therefore, Corollary 2 is proven.

\section{H Proof of Lemma 2}

Proof: According to Guo and Zhang (2012), $\bar{c}^{f}=\alpha\left(\theta_{h}-\theta_{l}\right)^{2}, q_{l}^{f}=\theta_{l}-\frac{\alpha\left(\theta-\theta_{l}\right)}{1-\alpha}$ for $c<\bar{c}$ and $q_{l}^{f}=\theta_{l}-\frac{c}{(1-\alpha)\left(\theta_{h}-\theta_{l}\right)}$ for $c \geq \bar{c}$. In the proof of Proposition 5, we will show that for $c \geq \bar{c}^{f}, q_{l}<q_{l}^{f}$. Therefore, the $c$ such that $q_{l}=q_{l}^{f}$ should be strictly below $\bar{c}^{f}$. For $c<\bar{c}$, both $q_{l}$ and $q_{l}^{f}$ take interior solutions, and the relative magnitude between $q_{l}$ and $q_{l}^{f}$ does not depend on $c$. Therefore, if we focus on the deliberation cost $c$ to make $q_{l}=q_{l}^{f}$, reasonably this $c$ should be considered for $c \geq \bar{c}$. For $\bar{c} \leq c<\bar{c}^{f}, q_{l}$ takes a boundary solution and $q_{l}^{f}$ takes an interior solution. In this situation, $q_{l} \geq q_{l}^{f}$ if and only if $c \leq \frac{1}{\beta} \frac{\alpha}{1-\alpha}\left(\theta_{h}-\theta_{l}\right)\left[\alpha\left(\beta^{h}-\beta\right) \theta_{h}+(1-\alpha)\left(\beta^{l}-\beta\right) \theta_{l}\right]$. Therefore, $c=\frac{1}{\beta} \frac{\alpha}{1-\alpha}\left(\theta_{h}-\theta_{l}\right)\left[\alpha\left(\beta^{h}-\beta\right) \theta_{h}+(1-\alpha)\left(\beta^{l}-\beta\right) \theta_{l}\right]$ is the deliberation cost threshold that makes $q_{l}=q_{l}^{f}$. According to its derivation, $\frac{1}{\beta} \frac{\alpha}{1-\alpha}\left(\theta_{h}-\theta_{l}\right)\left[\alpha\left(\beta^{h}-\beta\right) \theta_{h}+\right.$ $\left.(1-\alpha)\left(\beta^{l}-\beta\right) \theta_{l}\right]<\bar{c}^{f}$ for any $\alpha \in(0,1)$. However, for $\bar{c}<\frac{1}{\beta} \frac{\alpha}{1-\alpha}\left(\theta_{h}-\theta_{l}\right)\left[\alpha\left(\beta^{h}-\beta\right) \theta_{h}+(1-\alpha)\left(\beta^{l}-\beta\right) \theta_{l}\right]$, it requires that $\alpha>1-\frac{1}{\frac{\beta^{h}\left(\beta-\beta^{l}\right)}{\beta(1-\beta)}}$. Otherwise, if $\alpha \leq 1-\frac{1}{\frac{\beta^{h}\left(\beta-\beta^{l}\right)}{\beta(1-\beta)}}$, $\bar{c} \geq \frac{1}{\beta} \frac{\alpha}{1-\alpha}\left(\theta_{h}-\theta_{l}\right)\left[\alpha\left(\beta^{h}-\beta\right) \theta_{h}+(1-\alpha)\left(\beta^{l}-\beta\right) \theta_{l}\right]$. From this proof, note that for any $\alpha \in(0,1), q_{l}<q_{l}^{f}$ for $c>\frac{1}{\beta} \frac{\alpha}{1-\alpha}\left(\theta_{h}-\theta_{l}\right)\left[\alpha\left(\beta^{h}-\beta\right) \theta_{h}+(1-\alpha)\left(\beta^{l}-\beta\right) \theta_{l}\right]$. Therefore, Lemma 2 is proven.

\section{Proof of Proposition 5}

Proof: According to the Proof of Lemma 2, for any $\alpha \in(0,1), q_{l}<q_{l}^{f}$ for $c>\frac{1}{\beta} \frac{\alpha}{1-\alpha}\left(\theta_{h}-\theta_{l}\right)\left[\alpha\left(\beta^{h}-\right.\right.$ $\left.\beta) \theta_{h}+(1-\alpha)\left(\beta^{l}-\beta\right) \theta_{l}\right]$. Therefore, in this proof, we focus on proving the comparison between $q_{l}$ and $q_{l}^{f}$ for $c \leq \frac{1}{\beta} \frac{\alpha}{1-\alpha}\left(\theta_{h}-\theta_{l}\right)\left[\alpha\left(\beta^{h}-\beta\right) \theta_{h}+(1-\alpha)\left(\beta^{l}-\beta\right) \theta_{l}\right]$.

According to Lemma 1 , it is known that $\bar{c}<\bar{c}^{f}$. Thus, for $c<\bar{c}$, both $q_{l}$ and $q_{l}^{f}$ are interior solutions of respective product line design problems. In this situation, $q_{l}>q_{l}^{f}$ if 1$)$ given that $1>\frac{\beta^{h}\left(\beta-\beta^{l}\right)}{\beta(1-\beta)}$, $\alpha \in\left(\frac{\frac{\beta^{h}\left(\beta-\beta^{l}\right)}{\beta(1-\beta)}}{1+\frac{\beta^{h}\left(\beta-\beta^{l}\right)}{\beta(1-\beta)}}, 1\right)$, or 2$)$ given that $1<\frac{\beta^{h}\left(\beta-\beta^{l}\right)}{\beta(1-\beta)}$, $\alpha \in\left(\max \left\{1-\frac{1}{\frac{\beta^{h}\left(\beta-\beta^{l}\right)}{\beta(1-\beta)}}, \frac{\frac{\beta^{h}\left(\beta-\beta^{l}\right)}{\beta(1-\beta)}}{1+\frac{\beta^{h}\left(\beta-\beta^{l}\right)}{\beta(1-\beta)}}\right\}, 1\right)$. The cases imply that for $c \leq \bar{c}, q_{l}>q_{l}^{f}$ if $\alpha \in\left(\max \left\{1-\frac{1}{\frac{\beta^{h}\left(\beta-\beta^{l}\right)}{\beta(1-\beta)}}, \frac{\frac{\beta^{h}\left(\beta-\beta^{l}\right)}{\beta(1-\beta)}}{1+\frac{\beta^{h}\left(\beta-\beta^{l}\right)}{\beta(1-\beta)}}\right\}, 1\right)$.

Given that $\alpha>1-\frac{1}{\frac{\beta^{h}\left(\beta-\beta^{l}\right)}{\beta(1-\beta)}}$, from Lemma 2, it is known that in this situation, $\bar{c}<\frac{1}{\beta} \frac{\alpha}{1-\alpha}\left(\theta_{h}-\theta_{l}\right)\left[\alpha\left(\beta^{h}-\right.\right.$ $\left.\beta) \theta_{h}+(1-\alpha)\left(\beta^{l}-\beta\right) \theta_{l}\right]$. In this situation, according to the Proof of Lemma $2, q_{l}$ takes the boundary solution while $q_{l}^{f}$ takes the interior solution of each product line design problem. Thus, $q_{l} \geq q_{l}^{f}$ if and only if $c \leq$ $\frac{1}{\beta} \frac{\alpha}{1-\alpha}\left(\theta_{h}-\theta_{l}\right)\left[\alpha\left(\beta^{h}-\beta\right) \theta_{h}+(1-\alpha)\left(\beta^{l}-\beta\right) \theta_{l}\right]$. Therefore, in conclusion, for $\alpha \in\left(\max \left\{1-\frac{1}{\frac{\beta^{h}\left(\beta-\beta^{l}\right)}{\beta(1-\beta)}}, \frac{\frac{\beta^{h}\left(\beta-\beta^{l}\right)}{\beta(1-\beta)}}{1+\frac{\beta^{h}\left(\beta-\beta^{l}\right)}{\beta(1-\beta)}}\right\}, 1\right)$, $q_{l} \geq q_{l}^{f}$ if $c \leq \frac{1}{\beta} \frac{\alpha}{1-\alpha}\left(\theta_{h}-\theta_{l}\right)\left[\alpha\left(\beta^{h}-\beta\right) \theta_{h}+(1-\alpha)\left(\beta^{l}-\beta\right) \theta_{l}\right]$. Therefore, Proposition 5 is proven.

\section{J Proof of Proposition 6}

Proof: According to equation 3 and 4, $\triangle p=$ $\left[\frac{\alpha \beta^{h}}{\beta} \theta_{h}+\frac{(1-\alpha) \beta^{l}}{\beta} \theta_{l}\right] \Delta q-\frac{c}{\beta}$. For $c<\bar{c}, \Delta q=\phi\left(\theta_{h}-\theta_{l}\right)$. In this situation, $\Delta p>0$ can be transformed to the following inequality with respect to $\theta_{h}: \alpha \beta^{h} \theta_{h}^{2}+[(1-$ $\left.\alpha) \beta^{l}-\alpha \beta^{h}\right] \theta_{l} \theta_{h}-\left[(1-\alpha) \beta^{l} \theta_{l}^{2}+\frac{c}{\phi}\right]>0$. For $\theta_{h}>$ $\frac{-\left[(1-\alpha) \beta^{l}-\alpha \beta^{h}\right] \theta_{l}+\sqrt{\left\{\left[(1-\alpha) \beta^{l}-\alpha \beta^{h}\right]^{2}+4 \alpha(1-\alpha) \beta^{h} \beta^{l}\right\} \theta_{l}^{2}+\frac{4 \alpha \beta^{h}}{\phi} c}}{2 \alpha \beta^{h}}$,

or

$\theta_{h}<\frac{-\left[(1-\alpha) \beta^{l}-\alpha \beta^{h}\right] \theta_{l}-\sqrt{\left\{\left[(1-\alpha) \beta^{l}-\alpha \beta^{h}\right]^{2}+4 \alpha(1-\alpha) \beta^{h} \beta^{l}\right\} \theta_{l}^{2}+\frac{4 \alpha \beta^{h}}{\phi} c}}{2 \alpha \beta^{h}}$, $\alpha \beta^{h} \theta_{h}^{2}-\left[\alpha \beta^{h}-(1-\alpha) \beta^{l}\right] \theta_{l} \theta_{h}-\left[\begin{array}{ll}1 & -\end{array}\right.$ $\left.\alpha) \beta^{l} \theta_{l}^{2}+\frac{c}{\phi}\right]>0$ is held. However, $\frac{-\left[(1-\alpha) \beta^{l}-\alpha \beta^{h}\right] \theta_{l}-\sqrt{\left\{\left[(1-\alpha) \beta^{l}-\alpha \beta^{h}\right]^{2}+4 \alpha(1-\alpha) \beta^{h} \beta^{l}\right\} \theta_{l}^{2}+\frac{4 \alpha \beta^{h}}{\phi} c}}{2 \alpha \beta^{h}}<$

0. Therefore, for $c<\bar{c}, \triangle \Delta^{2}>0$ if and only if $\theta_{h}>$ $\frac{-\left[(1-\alpha) \beta^{l}-\alpha \beta^{h}\right] \theta_{l}+\sqrt{\left\{\left[(1-\alpha) \beta^{l}-\alpha \beta^{h}\right]^{2}+4 \alpha(1-\alpha) \beta^{h} \beta^{l}\right\} \theta_{l}^{2}+\frac{4 \alpha \beta^{h}}{\phi} c}}{2 \alpha \beta^{h}}$.

For $c \geq \bar{c}, \Delta p=\left[\frac{\alpha \beta^{h} \theta_{h}+(1-\alpha) \beta^{l} \theta_{l}}{\alpha\left(\beta^{h}-\beta\right) \theta_{h}+(1-\alpha)\left(\beta^{l}-\beta\right) \theta_{l}}-1\right] \frac{c}{\beta}$. Because $\frac{\alpha \beta^{h} \theta_{h}+(1-\alpha) \beta^{l} \theta_{l}}{\alpha\left(\beta^{h}-\beta\right) \theta_{h}+(1-\alpha)\left(\beta^{l}-\beta\right) \theta_{l}}>1$ is always held, therefore for $c \geq \bar{c}, \triangle p>0$. 


\section{K Proof of Lemma 3}

Proof: For $c<\bar{c}, q_{l}=\frac{\alpha \beta^{h}\left(\beta^{l}-\beta\right)}{\beta(1-\beta)} \theta_{h}+\left[1-\frac{\alpha \beta^{h}\left(\beta^{l}-\beta\right)}{\beta(1-\beta)}\right] \theta_{l}$. In this case, it is straightforward that $q_{l}>0$ if and only if $\frac{\theta_{l}}{\theta_{h}}>-\frac{\frac{\alpha \beta^{h}\left(\beta^{l}-\beta\right)}{\beta(1-\beta)}}{1-\frac{\alpha \beta^{h}\left(\beta^{l}-\beta\right)}{\beta(1-\beta)}}$.

For $c \geq \bar{c}, q_{l}=\theta_{l}-\frac{\beta c}{\alpha\left(\beta^{h}-\beta\right) \theta_{h}+(1-\alpha)\left(\beta^{l}-\beta\right) \theta_{l}}$. $q_{l}>0$ indicates the following inequality with respect to $\theta_{l}:(1-\alpha)\left(\beta-\beta^{l}\right) \theta_{l}^{2}-\alpha\left(\beta^{h}-\beta\right) \theta_{h} \theta_{l}+\beta c<0$. Solving this inequality, we find that it is held for $\theta_{h}>\frac{2 \sqrt{(1-\alpha)\left(\beta-\beta^{l}\right) \beta c}}{\alpha\left(\beta^{h}-\beta\right)}$ and $\frac{2\left(\beta^{h}-\beta\right) \theta_{h}-\sqrt{\alpha^{2}\left(\beta^{h}-\beta\right)^{2} \theta_{h}^{2}-4(1-\alpha)\left(\beta-\beta^{l}\right) \beta c}}{2(1-\alpha)\left(\beta-\beta^{l}\right)}<\theta_{l}<$ $\frac{2\left(\beta^{h}-\beta\right) \theta_{h}+\sqrt{\alpha^{2}\left(\beta^{h}-\beta\right)^{2} \theta_{h}^{2}-4(1-\alpha)\left(\beta-\beta^{l}\right) \beta c}}{2(1-\alpha)\left(\beta-\beta^{l}\right)}$.

Lemma 3 is proven.

Therefore,

\section{Proof of Proposition 7}

Proof: For $c<\bar{c}, \theta_{l} q_{l}-p_{l}=\frac{\alpha \beta^{h} \beta^{l}}{\beta}\left(\theta_{h}-\theta_{l}\right)^{2} \phi-\frac{\beta^{l} c}{\beta}$. Therefore, $\theta_{l} q_{l}-p_{l}>0$ if and only if $\theta_{h}-\theta_{l}>\sqrt{\frac{c}{\alpha \beta^{h} \phi}}$.

For $c \geq \bar{c}, \theta_{l} q_{l}-p_{l}=c \frac{\beta^{l}}{\beta} \frac{\alpha \beta\left(\theta_{h}-\theta_{l}\right)}{\alpha\left(\beta^{h}-\beta\right) \theta_{h}+(1-\alpha)\left(\beta^{l}-\beta\right) \theta_{l}}$, which is always greater than zero. Therefore, for $c \geq \bar{c}, \theta_{l} q_{l}-p_{l}>0$ is always held.

\section{Proof of Proposition 8 and Corollary 4}

Proof: $\pi_{h}=p_{h}-\frac{q_{h}^{2}}{2}=A_{h} q_{h}+B_{h} q_{l}-\frac{\left(1-\beta^{l}\right) c}{\beta}-\frac{q_{h}^{2}}{2}$. Therefore, $\pi_{h}>0$ can be viewed as an inequality with respect to $q_{h}: \frac{q_{h}^{2}}{2}-A_{h} q_{h}-B_{h} q_{l}+\frac{\left(1-\beta^{l}\right) c}{\beta}<0$. Solving this inequality, we obtain that $0<A_{h}-\sqrt{A_{h}^{2}+\left(2 B_{h} q_{l}-\frac{1-\beta^{l}}{2} c\right)}<$ $q_{h}<A_{h}+\sqrt{A_{h}^{2}+\left(2 B_{h} q_{l}-\frac{1-\beta^{l}}{2} c\right)}$ and $q_{l}<$ $\frac{\left[\frac{\alpha \beta^{h}\left(1-\beta^{l}\right)}{\beta} \theta_{h}+\beta^{l}\left[1+\frac{(1-\alpha)\left(1-\beta^{l}\right)}{\beta}\right] \theta_{l}\right]^{2}-\frac{\left(1-\beta^{l}\right) c}{2}}{2\left(\theta_{h}-\theta_{l}\right) \frac{\alpha \beta^{h}\left(1-\beta^{l}\right)}{\beta}} . \quad$ We require that $0<c<\frac{2\left[\frac{\alpha \beta^{h}\left(1-\beta^{l}\right)}{\beta} \theta_{h}+\beta^{l}\left[1+\frac{(1-\alpha)\left(1-\beta^{l}\right)}{\beta}\right] \theta_{l}\right]^{2}}{1-\beta^{l}}$ to ensure $q_{l}>0$. Therefore, Proposition 8 is proven.

$$
\begin{gathered}
\text { Because for } c \geq \frac{2\left[\frac{\alpha \beta^{h}\left(1-\beta^{l}\right)}{\beta} \theta_{h}+\beta^{l}\left[1+\frac{(1-\alpha)\left(1-\beta^{l}\right)}{\beta}\right] \theta_{l}\right]^{2}}{1-\beta^{l}}, \\
\frac{\left[\frac{\alpha \beta^{h}\left(1-\beta^{l}\right)}{\beta} \theta_{h}+\beta^{l}\left[1+\frac{(1-\alpha)\left(1-\beta^{l}\right)}{\beta}\right] \theta_{l}\right]^{2}-\frac{\left(1-\beta^{l}\right) c}{2}}{2\left(\theta_{h}-\theta_{l}\right) \frac{\alpha \beta^{h}\left(1-\beta^{l}\right)}{\beta}} \leq 0 . \quad \text { In }
\end{gathered}
$$

this situation, any $q_{l}>0$ can satisfy that $q_{l}>$ $\frac{\left[\frac{\alpha \beta^{h}\left(1-\beta^{l}\right)}{\beta} \theta_{h}+\beta^{l}\left[1+\frac{(1-\alpha)\left(1-\beta^{l}\right)}{\beta}\right] \theta_{l}\right]^{2}-\frac{\left(1-\beta^{l}\right) c}{2}}{2\left(\theta_{h}-\theta_{l}\right) \frac{\alpha \beta^{h}\left(1-\beta^{l}\right)}{\beta}}$ and there is no solution for the inequality $\frac{q_{h}^{2}}{2}-A_{h} q_{h}-B_{h} q_{l}+\frac{\left(1-\beta^{l}\right) c}{\beta}<0$. Therefore, for $c \geq \frac{2\left[\frac{\alpha \beta^{h}\left(1-\beta^{l}\right)}{\beta} \theta_{h}+\beta^{l}\left[1+\frac{(1-\alpha)\left(1-\beta^{l}\right)}{\beta}\right] \theta_{l}\right]^{2}}{1-\beta^{l}}$, $\pi_{h} \leq 0$. Therefore, Corollary 4 is proven.

\section{N Proof of Proposition 9}

Proof: $\pi_{l}=p_{l}-\frac{q_{l}^{2}}{2}=A_{l} q_{h}+B_{l} q_{l}+\frac{\beta^{l} c}{\beta}-\frac{q_{l}^{2}}{2}$. Therefore, $\pi_{l}>0$ can be viewed as an inequality with respect to $q_{l}$ : $\frac{q_{l}^{2}}{2}-B_{l} q_{l}-A_{l} q_{h}-\frac{\beta^{l} c}{\beta}<0$. Solving this inequality, we obtain that $B_{l}-\sqrt{B_{l}^{2}+2\left[A_{l} q_{h}+\frac{\beta^{l}}{\beta} c\right]}<q_{l}<$ $B_{l}+\sqrt{B_{l}^{2}+2\left[A_{l} q_{h}+\frac{\beta^{l}}{\beta} c\right]}$ and $q_{h}<-\frac{B_{l}^{2}+2 \frac{\beta^{l}}{\beta} c}{2 A_{l}}$. Note that $-\frac{B_{l}^{2}+2 \frac{\beta^{l}}{\beta} c}{2 A_{l}}>0$ for any $c>0$. Therefore, Proposition 9 is proven.

\section{REFERENCES}

[1] Kahneman D (1973) Attention and Effort (Englewood Cliffs, NJ: Prentice Hall).

[2] Becker G, DeGroot M and Marschak J (1963) Stochastic Models of Choice Behavior. Behavioral Sci. 8: 41-55.

[3] Bolton P and M Dewatripont (2005). Contract Theory (the MIT Press).

[4] Buttler D J and Loomes G C (2007) Imprecision as an Account of the Preference Reversal Phenomenon. Amer. Econom. Rev. 97 (1): 277 - 297.

[5] Cubitt R P, Navarro-Martinez D, and Starmer C (2015) On Preference Imprecision. J. Risk and Uncertainty. 50 (1): 1 - 34.

[6] Fechner G (1860, reprinted in 1966) Elements of Psychophysics (New York: Holt, Rinehart and Winston).

[7] Fudenberg D, Strack P and T Strzalecki (2015) Speed, Accuracy, and the Optimal Timing of Choices. Working Paper. Massachusetts Institute of Technology.

[8] Guo L and J Zhang (2012) Consumer Deliberation and Product Line Design. Marketing Sci. 31 (6): 995-1007.

[9] Harless D and Camerer C (1994) The Predictive Utility of Generalized Expected Utility Theories. Econometrica. 62: 12511289.

[10] Hey J and Orme C (1994) Investigating Generalizations of Expected Utility Theory Using Experimental Data. Econometrica. 62: 1291-1326.

[11] Kamenica E (2008) Contextual Inference in Markets: On the Informational Content of Product Lines. Amer. Econom. Rev. 98 (5): 2127-2149.

[12] Lachman R, Lachman J L, and B C Butterfield (1979, reprinted in 2015) Cognitive Psychology and Information Processing: an Introduction (Psychology Press, Taylor \& Francis Group, London and New York).

[13] Lewis T and D Sappington (1993) Ignorance in Agency Problems. J. Econom. Theory. 61: 169-183.

[14] Lewis T and D Sappington (1997) Information Management in Incentive Problems. J. Political Economy. 105 (4): 796-821. 
[15] Loomes G and Sugden R (1995) Incorporating a Stochastic Element into Decision Theories. Euro. Econom. Rev. 39: 641-648.

[16] Loomes G (2005) Modelling the Stochastic Component of Behavior in Experiments: Some Issues for the Interpretation of Data. Experimental Econom. 8 (4): 301-323.

[17] Lumpkin S (2010) Consumer Protection and Financial Innova- tion: A Few Basic Propositions. OECD Journal: Financial Market Trends. 2010 (1). OECD 2010.

[18] World Bank Group (2017) Good Practices for Financial Consumer Protection. 2017 International Bank for Reconstruction and Development / The World Bank Group. 